\title{
Intake of total and added sugars and nutrient dilution in Australian children and adolescents
}

\author{
Jimmy Chun Yu Louie ${ }^{1,2_{*}}$ and Linda C. Tapsell ${ }^{1}$ \\ ${ }^{1}$ School of Medicine, Faculty of Science, Medicine and Health, The University of Wollongong, Wollongong, NSW 2522, \\ Australia \\ ${ }^{2}$ School of Molecular Bioscience, Faculty of Science, The University of Sydney, Sydney, NSW 2006, Australia \\ (Submitted 20 April 2015 - Final revision received 28 June 2015 - Accepted 12 August 2015 - First published online 28 September 2015)
}

\section{Abstract}

This analysis aimed to examine the association between intake of sugars (total or added) and nutrient intake with data from a recent Australian national nutrition survey, the 2007 Australian National Children's Nutrition and Physical Activity Survey (2007ANCNPAS). Data from participants ( $n$ 4140; $51 \%$ male) who provided $2 \times$ plausible $24-\mathrm{h}$ recalls were included in the analysis. The values on added sugars for foods were estimated using a previously published ten-step systematic methodology. Reported intakes of nutrients and foods defined in the 2007ANCNPAS were analysed by age- and sex-specific quintiles of \%energy from added sugars (\%EAS) or \%energy from total sugars (\%ETS) using ANCOVA. Linear trends across the quintiles were examined using multiple linear regression. Logistic regression analysis was used to calculate the OR of not meeting a specified nutrient reference values for Australia and New Zealand per unit in \%EAS or \%ETS. Analyses were adjusted for age, sex, BMI $z$-score and total energy intake. Small but significant negative associations were seen between \%EAS and the intakes of most nutrient intakes (all $P<0 \cdot 001$ ). For \%ETS the associations with nutrient intakes were inconsistent; even then they were smaller than that for \%EAS. In general, higher intakes of added sugars were associated with lower intakes of most nutrient-rich, 'core' food groups and higher intakes of energy-dense, nutrient-poor 'extra' foods. In conclusion, assessing intakes of added sugars may be a better approach for addressing issues of diet quality compared with intakes of total sugars.

\section{Key words: Total sugars: Added sugars: Nutrient dilution: Australian children and adolescents}

The role of sugars in the modern diet has always been a controversial topic ${ }^{(1-9)}$. 'Sugar' is a term that can refer to a food mostly used in a culinary sense, an ingredient in prepared foods and beverages such as bakery items and soft drinks, or to a set of compounds (sugars) naturally occurring as part of the carbohydrate component of mostly plant foods (e.g. fructose in fruit and lactose in milk). A 'total sugars' value is a problematic marker of diet quality ${ }^{(10)}$ because this reflects the sugar content of nutritious foods such as fruit and dairy products, which contain naturally occurring sugars, as well as added or culinary sugar, which serves only to add energy ( $\mathrm{kcal} / \mathrm{kJ})$ without concomitant nutrients. Public health agencies have been suggesting for some time that people limit or moderate their intake of added sugars to reduce their total energy intake $(\mathrm{EI})^{(11-13)}$. In support of these recommendations, a recent systematic review ${ }^{(14)}$ has concluded that in ad libitum conditions, increased added sugar consumption is associated with a $0.75 \mathrm{~kg}$ weight gain.

High diet quality remains the target for health, and this is evaluated in terms of nutrients delivered for a given energy value. There is some concern that the sugar content may dilute diet quality, that is, the more sugar, the less nutrients for a given energy value. However, the evidence regarding the association between sugar intake (total or added) and nutrient intake is less consistent ${ }^{(15-19)}$. This may be due to the different use of analytical methods, including different methods for energy adjustment ${ }^{(20)}$. To date, no study had found that a higher intake of added sugars is associated with improved nutrient intake after adjusting for total EI, and this suggests that the sugar content of the diet may be contributing to nutrient dilution.

Previous analyses of the 1995 Australian national nutrition survey ${ }^{(15,19)}$ revealed that in children and adolescents a higher added/refined sugar intake was associated with poorer nutrient intake. However, recent data suggest that the apparent consumption of refined sugars in Australia has declined in recent years ${ }^{(1,21)}$. Using a more up-to-date data set from the 2007 Australian National Children's Nutrition and Physical Activity Survey (2007ANCNPAS) ${ }^{(22)}$, the aim of our study was to test whether a higher total or added sugar intake was associated with nutrient dilution (where 'sugar' refers to common chemical forms of sugar). We hypothesise that a higher intake of added

Abbreviations: 2007 ANCNPAS, the 2007 Australian National Children's Nutrition and Physical Activity Survey; EI, energy intake; \%EAS, \%energy from added sugars; \%ETS, \%energy from total sugars; NRV, nutrient reference values; PAL, physical activity level.

* Corresponding author: Dr J. C. Y. Louie, fax +61 28627 1605, email jimmy.louie@sydney.edu.au 
sugar but not total sugar is consistently associated with nutrient dilution in the diets of Australian children and adolescents.

\section{Methods \\ The 2007 Australian National Children's Nutrition and Physical Activity Survey}

The 2007 ANCNPAS was commissioned in 2007 by the Australian Government Department of Agriculture, Fisheries and Forestry and the Australian Food and Grocery Council ${ }^{(22)}$. The methodology of the 2007ANCNPAS has been described in detail elsewhere ${ }^{(23)}$. In brief, the survey measured the dietary intakes of food and beverages as well as use of supplements using two multiple-pass 24-h recalls collected 7-21 d apart. These data were collected on children aged $2-16$ years ( $n$ 4834) between 22 February and 30 August 2007. Dietary data were collected from the primary-care giver on children aged 2-8 years; children aged 9 years and older reported their own dietary intake. Nutrient intake from supplements was not considered in the current study.

\section{Added sugars analyses}

Dietary intake data were entered into a purpose-built database, with nutrition compositions based on the AUSNUT2007 database $^{(24)}$. Using this database, the content of added sugars, defined as all refined sugars added during cooking or manufacturing, of foods was estimated on the basis of a ten-step methodology previously published ${ }^{(25)}$. Briefly, the ten steps are as follows:

Step 1: Assign $0 \mathrm{~g}$ added sugar to foods with $0 \mathrm{~g}$ total sugars. Step 2: Assign $0 \mathrm{~g}$ added sugar to foods identified as noadded-sugar food groups.

Step 3: Assign $100 \%$ of total sugars as added sugar for foods in $100 \%$-added-sugar food groups.

Step 4: Calculate added sugar value on the basis of standard recipe used in the food composition database - proportioning method where added sugar contents of ALL ingredients were available from steps $1-3$.

Step 5: Calculate added sugar value on the basis of comparison to values from unsweetened variety.

Step 6: Decide on proportion of added sugar on the basis of analytical data.

Step 7: Use borrowed values from similar products from steps 1 to 6 or from overseas.

Step 8: Subjectively estimate added sugar content on the basis of ingredients and/or common recipes (e.g. obtained from popular recipe books).

Step 9: Calculate added sugar content on the basis of standard recipe, which includes ingredients with values assigned at steps 5-8, using the proportioning method.

Step 10: Assign $50 \%$ of total sugars as added sugars.

More details about the steps could be found in the original paper $^{(25)}$.

\section{Data cleaning}

Data from children who completed only one 24-h recall ( $n$ 179) were excluded from the analyses. The plausibility of data from the remaining sample was assessed using the Goldberg cut-off for specific physical activity level (PAL) ${ }^{(26)}$. A default PAL of 1.55 was assigned to children aged 8 years or below as no physical activity data were available. We excluded data from 339 extreme under-reporters (EI:BMR ratio: 0.3-1.5; PAL: $1 \cdot 2-2 \cdot 8 ; 47 \%$ male) and 129 extreme over-reporters (EI:BMR ratio: $2 \cdot 2-4 \cdot 7$; PAL: $1 \cdot 1-2 \cdot 3 ; 51 \%$ male) on the basis of this method. An additional forty-seven subjects were excluded because weight and/or height were not recorded and as the plausibility of the data could not be assessed. The final data set included data from 4140 participants ( $51 \%$ male) who provided $2 \times 24-h$ recalls.

\section{Comparison to the nutrient reference values of Australia and New Zealand}

Usual intakes of energy and macro- and micronutrients were calculated using the multiple source method (MSM) ${ }^{(27)}$ to account for intra-personal variability. The usual nutrient intakes of the participants were compared with the latest Nutrient reference values (NRV) for Australia and New Zealand ${ }^{(28)}$ using criteria relevant to available standards. Thus, for the group of nutrients with an estimated average requirement (EAR) (Ca, Fe, I, Zn, Mg, P, vitamin A (as retinol equivalents), thiamin, riboflavin, dietary folate equivalents and vitamin $\mathrm{C}$ ), intakes lower than the EAR were considered not meeting the NRV. For nutrients with an adequate intake (AI) value (K, long chain $n-3$ PUFA (LC $n-3$ PUFA), dietary fibre, vitamin D and vitamin E), intakes lower than the AI were considered not meeting the NRV. Upper limits and suggested targets were considered for nutrients known to be consumed in excess. Thus, intakes higher than the upper level (UL) for $\mathrm{Na}$ were considered not meeting the NRV, and EI from SFA $>10 \%$ were considered not meeting the SFA NRV.

\section{Comparison with food pattern intakes}

The values for intakes of sugars were compared with reported intakes of food groups reported in the AUSNUT2007 database. Nutrient-rich, core and discretionary 'extra' foods were referred to as defined by the Australian Dietary Guidelines ${ }^{(29)}$ and Rangan et al. ${ }^{(30,31)}$. In brief, core foods included fruits, most vegetables, legumes, nuts, seeds, eggs, fish, most meats and poultry and most dairy foods; and 'extras' were nutrient-poor foods and beverages. A detailed list of how food groups were classified is available as online Supplementary Table S1.

\section{Statistical analysis}

Data were weighted to account for over- or under-sampling to enable representation of the Australian population aged 2-16 years in terms of age group, sex and region. BMI $z$-scores of the subjects were calculated using the WHO Anthro SPSS macro (version 3.1, June 2010). Intakes of nutrients and foods from broad food groups used in the 2007ANCNPAS ${ }^{(23)}$ by age- and sex-specific quintiles of usual \%energy from added sugars (\%EAS) or \%energy from total sugars (\%ETS) were calculated using ANCOVA, adjusted for BMI $z$-score and total EI. The linear trends across the quintiles were examined using multiple 
linear regression, with \%EAS or \%ETS as a continuous independent variable and the nutrient intake as a continuous dependent variable, adjusted for age, sex, BMI $z$-score and total EI.

Pearson's $\chi^{2}$ test was used to test for difference in the proportion of subjects not meeting the NRV for Australia and New Zealand across the quintiles. Logistic regression analysis was used to calculate the OR of not meeting the NRV for Australia and New Zealand per unit increase in \%EAS or \%ETS, adjusted for age, sex, BMI $z$-score and total EI. For the NRV comparisons with \%EAS as the independent variable, sources of added sugars were further stratified as from 'core' foods or from 'extra' foods.

To test whether the data collection method - namely, parental $v$. self-report - may have biased the findings, sensitivity analyses were performed where the sample was stratified into 8 years old or below (parental report) and 9 years old or above (self-report).

Values were presented as means and $95 \%$ CI for continuous variables and as percentages for categorical variables. Because a large number of tests were conducted, $P<0.01$ was considered to indicate marginal statistical significance, and $P<0.001$ was considered significant to reduce the chance of type I error. All statistical analyses were carried out using Statistical Packages for Social Science version 22.0 (IBM Corporation, 2010)

\section{Results}

Values for usual intake of added sugars (as \%EAS) were negatively associated with all nutrient intakes (all $P_{\text {trend }}<0 \cdot 001$ ) except of course for total carbohydrate and sugar intake, which were positively associated $(P<0.001)$. It was also associated with a slight increase in EI $\left(\beta: 17.7\right.$ (SE 5.0); $\left.P_{\text {trend }}<0.001\right)$. The association with SFA (absolute or expressed as \% of energy) intake was not significant (Table 1). The associations were of small magnitude (a change of approximately $1 \%$ or less in intake per unit increase in \%EAS). Similar results were found for associations with \%ETS (Table 2), but the magnitude of association was even smaller than that for \%EAS in most cases except $\mathrm{Na}$, and the direction of associations was less consistent. There was no linear relationship between total EI and \%ETS.

When the associations between intakes of total and added sugars and likelihood of meeting the NRV for Australia and New Zealand were examined (Tables 3 and 4), a higher \%EAS was consistently associated with poorer micronutrient intake (all $\left.P_{\text {trend }}<0.001\right)$ except vitamin $\mathrm{C}\left(P_{\text {trend }}=0.103\right)$ and $\mathrm{Na}$ $\left(P_{\text {trend }}=0.082\right)$. For each unit increase in \%EAS, the increase in risk for not meeting the NRV for Australia and New Zealand ranged from $5 \%$ for I to $37 \%$ for riboflavin. Stratifying the source of added sugars revealed that this negative relationship between \%EAS and likelihood of not meeting the NRV only existed for added sugars from 'extra' food in most cases. The association between \%ETS and likelihood of not meeting the NRV is less consistent in direction. A higher \%ETS was associated with a greater likelihood of not meeting the NRV for LC $n-3$ PUFA, Fe and vitamin $\mathrm{E}$ and a lesser likelihood of not meeting the NRV for vitamin C, K, I and Na (all $P_{\text {trend }}<0.001$ ).

In general, higher intakes of added sugars were associated with lower intakes of most 'core' foods and a concurrent increase in most 'extra' foods (Table 5). The biggest reductions in 'core' foods ( $\beta$ (SE) per unit increase in \%EAS; all $\left.P_{\text {trend }}<0.001\right)$ observed were for non-alcoholic beverages $(-18.4$ (SE 2.5) g), cereal grains and cereal products $(-12.9$ (SE 0.8) g), fruit and fruit-based products $(-10.9$ (SE 0.8) g), dairy

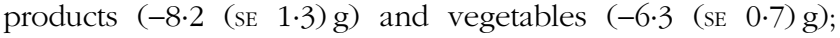
however, the biggest increases in 'extra' foods observed (all $\left.P_{\text {trend }}<0.001\right)$ were for sugar-sweetened beverages 43.7 (SE 1.2) g), dairy products (6.1 (SE 0.5) g), confectionery (3.9 (SE 0.2) g) and cereal-based products (3.6 (sE 0.6) g). Total sugar intake was positively associated with both 'core' and 'extra' dairy products, fruits, fruit juices, sugar-sweetened beverages, sugars and confectionery but negatively associated with meat, seafood, eggs, seeds and nuts, legumes, 'extra' vegetables and savoury snacks (Table 6 ; all $P_{\text {trend }}<0.001$ ).

Sensitivity analyses (see online Supplementary Tables S2-S13) revealed that the associations between usual intakes of added or total sugars and that of macro- and micronutrients were consistent between subjects with parental reports of dietary intake and those who self-reported their dietary intake. However, the magnitude of the association was generally larger among respondents who self-reported their intake, as shown by the larger $\beta(\mathrm{sE})$. The same is true for the association between $\%$ EAS and intake of food groups. More inconsistencies were observed when the likelihood of not meeting the NRV and intake of food groups were assessed according to \%ETS quintiles. Nonetheless, these observed inconsistencies were generally minor and, for the NRV analysis, were likely a result of reduced sample size for this analysis.

\section{Discussion}

This analysis supports the hypothesis that a higher intake of added sugars, but not total sugars, is associated with nutrient dilution in the diets of Australian children and adolescents. In addition, this association was only observed for added sugars from 'extra' foods in most cases. Using a more recent data set, our results confirm previous findings by Cobiac et $a l .{ }^{(19)}$ that the intake of total sugars is poorly correlated with micronutrient intake of Australian children and adolescents. Our results on added sugars are similar to other studies on children and adolescents around the world. In one study involving 1035 Irish children and adolescents using a 7-d weighed food record, Joyce \& Gibney $^{(18)}$ found that a higher added sugar consumption was associated with decreased dietary density of $\mathrm{Mg}, \mathrm{Ca}$, $\mathrm{Zn}$, vitamin $\mathrm{B}_{12}$ and vitamin $\mathrm{C}$, as well as an increased likelihood of intake below recommendations for some nutrients such as folate and $\mathrm{Zn}$, depending on age and sex. Here, patterns of intakes from different food groups were similar to those observed by us, for example, the negative association between decreased milk intake and added sugar intake. In another study on 1688 British children and adolescents, Gibson \& Boyd ${ }^{(32)}$ found that higher intakes of added sugar were negatively associated with intakes of most nutrients (as \% reference nutrient intake), where the reductions ranged from 14 to $24 \%$. They also found that subjects with higher \%EAS were more likely to have intakes below the EAR, and that subjects with a higher \%EAS had lower intakes of most core foods. 
Table 1. Mean* intake† of energy and nutrient according to age- and sex-specific quintiles of percentage energy (\%E) from added sugars (Mean values and $95 \%$ confidence intervals; $\beta$ coefficients with their standard errors)

\begin{tabular}{|c|c|c|c|c|c|c|c|c|c|c|c|c|c|c|}
\hline \multirow[b]{3}{*}{ Nutrients } & \multicolumn{10}{|c|}{$\%$ from added sugars } & \multirow[b]{3}{*}{$\beta \ddagger$} & \multirow[b]{3}{*}{ SE } & \multirow[b]{3}{*}{$R^{2}$} & \multirow[b]{3}{*}{$P_{\text {trend }} \ddagger$} \\
\hline & \multicolumn{2}{|r|}{ Q1 } & \multicolumn{2}{|r|}{ Q2 } & \multicolumn{2}{|r|}{ Q3 } & \multicolumn{2}{|r|}{ Q4 } & \multicolumn{2}{|r|}{ Q5 } & & & & \\
\hline & Mean & $95 \% \mathrm{Cl}$ & Mean & $95 \% \mathrm{Cl}$ & Mean & $95 \% \mathrm{Cl}$ & Mean & $95 \% \mathrm{Cl}$ & Mean & $95 \% \mathrm{Cl}$ & & & & \\
\hline Added sugars (g) & $36 \cdot 1$ & $35 \cdot 6,36 \cdot 7$ & $47 \cdot 2$ & $46 \cdot 7,47 \cdot 8$ & $55 \cdot 8$ & $55 \cdot 3,56 \cdot 3$ & $65 \cdot 3$ & $64 \cdot 7,65 \cdot 8$ & 82.5 & $81 \cdot 9,83.0$ & 4.6 & 0.0 & 0.662 & $<0.001$ \\
\hline From core foods§ & 8.8 & $8.4,9.3$ & 11.3 & $10 \cdot 9,11 \cdot 7$ & $12 \cdot 3$ & $11.9,12 \cdot 7$ & $12 \cdot 4$ & $12 \cdot 0,12 \cdot 8$ & $13 \cdot 2$ & $12 \cdot 8,13.6$ & 0.4 & 0.0 & 0.112 & $<0.001$ \\
\hline From extra foods§ & $27 \cdot 6$ & $26 \cdot 8,28 \cdot 3$ & $36 \cdot 7$ & $36.0,37.5$ & $43 \cdot 7$ & $43 \cdot 0,44 \cdot 4$ & 53.9 & $52 \cdot 3,53 \cdot 7$ & $69 \cdot 0$ & $68 \cdot 2,68 \cdot 7$ & $4 \cdot 2$ & 0.0 & 0.860 & $<0.001$ \\
\hline$\%$ E added sugars & $7 \cdot 0$ & $6 \cdot 9,7 \cdot 1$ & 9.6 & $9.5,9.7$ & 11.4 & $11 \cdot 3,11.5$ & 13.5 & $13 \cdot 4,13 \cdot 6$ & $17 \cdot 2$ & $17 \cdot 1,17 \cdot 3$ & - & - & - & - \\
\hline From core foods§ & 1.9 & $1.8,1.9$ & $2 \cdot 3$ & $2 \cdot 3,2 \cdot 5$ & $2 \cdot 7$ & $2 \cdot 6,2 \cdot 7$ & $2 \cdot 7$ & $2 \cdot 6,2 \cdot 8$ & $2 \cdot 8$ & $2 \cdot 8,2 \cdot 9$ & 0.1 & 0.0 & 0.089 & $<0.001$ \\
\hline From extra foods§ & $5 \cdot 2$ & $5 \cdot 1,5 \cdot 4$ & $7 \cdot 3$ & $7 \cdot 1,7 \cdot 4$ & 8.8 & $8 \cdot 7,9 \cdot 0$ & $10 \cdot 8$ & $10 \cdot 7,11.0$ & $14 \cdot 2$ & $14 \cdot 1,14 \cdot 4$ & 0.9 & 0.0 & 0.842 & $<0.001$ \\
\hline Energy (kJ) & 7847.9 & $7721 \cdot 3,7974 \cdot 6$ & $8093 \cdot 7$ & $7966 \cdot 1,8221 \cdot 3$ & $8185 \cdot 6$ & $8058 \cdot 6,8312 \cdot 6$ & $8187 \cdot 5$ & $8061 \cdot 3,8313 \cdot 7$ & 8226.9 & $8100 \cdot 7,8353 \cdot 1$ & $17 \cdot 7$ & $5 \cdot 0$ & 0.589 & $<0.001$ \\
\hline Protein (g) & 84.6 & $83 \cdot 9,85 \cdot 2$ & $81 \cdot 6$ & $81 \cdot 0,82 \cdot 3$ & $80 \cdot 5$ & $79 \cdot 8,81 \cdot 1$ & $77 \cdot 7$ & $77 \cdot 0,78 \cdot 3$ & 73.7 & $73 \cdot 1,74 \cdot 4$ & $-1 \cdot 1$ & 0.0 & 0.797 & $<0.001$ \\
\hline$\%$ E protein & $17 \cdot 6$ & $17 \cdot 5,17 \cdot 8$ & $17 \cdot 1$ & $17 \cdot 0,17 \cdot 3$ & $16 \cdot 9$ & $16 \cdot 8,17 \cdot 0$ & $16 \cdot 4$ & $16 \cdot 3,16 \cdot 5$ & $15 \cdot 7$ & $15 \cdot 5,15 \cdot 8$ & -0.2 & 0.0 & 0.190 & $<0.001$ \\
\hline Total fat (g) & 68.9 & $68.5,69.4$ & $68 \cdot 3$ & $67.9,68.8$ & $68 \cdot 2$ & $67 \cdot 7,68 \cdot 7$ & $68 \cdot 2$ & $67 \cdot 7,68 \cdot 6$ & 66.4 & $65 \cdot 9,66 \cdot 9$ & -0.3 & 0.0 & 0.831 & $<0.001$ \\
\hline$\%$ E total fat & $31 \cdot 0$ & $30 \cdot 8,31 \cdot 3$ & $30 \cdot 7$ & $30 \cdot 4,31 \cdot 0$ & $30 \cdot 6$ & $30 \cdot 4,30 \cdot 8$ & $30 \cdot 6$ & $30 \cdot 4,30 \cdot 8$ & 299 & $29 \cdot 7,30 \cdot 1$ & -0.1 & 0.0 & 0.046 & $<0.001$ \\
\hline SFA (g) & $30 \cdot 4$ & $30 \cdot 1,30 \cdot 7$ & $30 \cdot 7$ & $30 \cdot 4,31 \cdot 0$ & $30 \cdot 8$ & $30.5,31 \cdot 2$ & $30 \cdot 9$ & $30 \cdot 6,31 \cdot 2$ & $30 \cdot 4$ & $30 \cdot 1,30 \cdot 7$ & -0.0 & 0.0 & 0.703 & 0.241 \\
\hline$\%$ E SFA & 13.7 & $13 \cdot 6,13 \cdot 9$ & $13 \cdot 8$ & $13.6,13.9$ & 13.9 & $13 \cdot 7,14.0$ & 13.9 & $13 \cdot 8,14.0$ & $13 \cdot 6$ & $13.5,13.8$ & -0.0 & 0.0 & 0.025 & 0.123 \\
\hline MUFA (g) & $24 \cdot 2$ & $24 \cdot 0,24 \cdot 4$ & $23 \cdot 8$ & $23 \cdot 6,24 \cdot 0$ & $23 \cdot 6$ & $23 \cdot 4,23 \cdot 8$ & $23 \cdot 7$ & $23 \cdot 5,23 \cdot 9$ & $23 \cdot 0$ & $22 \cdot 8,23 \cdot 2$ & -0.1 & 0.0 & 0.787 & $<0.001$ \\
\hline PUFA (g) & $9 \cdot 0$ & $8 \cdot 9,9 \cdot 1$ & $8 \cdot 8$ & $8.7,8.9$ & $8 \cdot 7$ & $8 \cdot 6,8 \cdot 8$ & $8 \cdot 6$ & $8.5,8.7$ & $8 \cdot 3$ & $8 \cdot 2,8 \cdot 4$ & -0.1 & 0.0 & 0.602 & $<0.001$ \\
\hline LC $n-3$ PUFA (mg) & $128 \cdot 1$ & $124 \cdot 6,131 \cdot 7$ & $120 \cdot 7$ & $117 \cdot 1,124 \cdot 3$ & $120 \cdot 3$ & $116 \cdot 8,123.9$ & $110 \cdot 7$ & $107 \cdot 1,114 \cdot 2$ & $103 \cdot 6$ & $100 \cdot 1,107 \cdot 2$ & -2.5 & 0.2 & 0.163 & $<0.001$ \\
\hline Total carbohydrate $(\mathrm{g})$ & 235.2 & $233 \cdot 9,236 \cdot 5$ & $241 \cdot 0$ & $239 \cdot 6,242 \cdot 3$ & 243.5 & $242 \cdot 2,244 \cdot 8$ & $247 \cdot 8$ & $246 \cdot 5,249 \cdot 1$ & $257 \cdot 2$ & $255 \cdot 9,258 \cdot 5$ & $2 \cdot 2$ & 0.1 & 0.888 & $<0.001$ \\
\hline$\%$ E carbohydrates & 49.9 & $49 \cdot 7,50 \cdot 2$ & $51 \cdot 1$ & $50 \cdot 8,51 \cdot 3$ & 51.5 & $51 \cdot 2,51 \cdot 7$ & $52 \cdot 2$ & $52 \cdot 0,52 \cdot 4$ & 53.9 & $53 \cdot 7,54 \cdot 1$ & 0.4 & 0.0 & 0.187 & $<0.001$ \\
\hline Total sugars (g) & $102 \cdot 4$ & $101 \cdot 2,103 \cdot 6$ & $112 \cdot 7$ & $111.5,113.9$ & $119 \cdot 3$ & $118 \cdot 1,120.5$ & $125 \cdot 0$ & $123 \cdot 8,126 \cdot 1$ & 139.7 & $138.5,140 \cdot 9$ & 3.6 & 0.1 & 0.729 & $<0.001$ \\
\hline$\% \mathrm{E}$ total sugars & $22 \cdot 0$ & $21 \cdot 7,22 \cdot 2$ & $24 \cdot 1$ & $23 \cdot 8,24 \cdot 3$ & $25 \cdot 4$ & $25 \cdot 2,25 \cdot 7$ & $26 \cdot 4$ & $26 \cdot 2,26 \cdot 7$ & $29 \cdot 3$ & $29 \cdot 1,29 \cdot 6$ & 0.7 & 0.0 & 0.450 & $<0.001$ \\
\hline Starch (g) & 129.6 & $128 \cdot 4,130.8$ & $126 \cdot 0$ & $124 \cdot 8,127 \cdot 2$ & $122 \cdot 2$ & $121 \cdot 0,123 \cdot 4$ & $121 \cdot 0$ & $119 \cdot 8,122 \cdot 2$ & 115.5 & $114 \cdot 3,116 \cdot 7$ & $-1 \cdot 3$ & 0.1 & 0.741 & $<0.001$ \\
\hline$\%$ E starch & $27 \cdot 2$ & $27 \cdot 0,27 \cdot 5$ & 26.5 & $26 \cdot 3,26 \cdot 8$ & $25 \cdot 6$ & $25 \cdot 4,25 \cdot 9$ & $25 \cdot 4$ & $25 \cdot 2,25 \cdot 7$ & $24 \cdot 3$ & $24 \cdot 0,24 \cdot 5$ & -0.3 & 0.0 & 0.115 & $<0.001$ \\
\hline Fibre $(\mathrm{g})$ & $22 \cdot 3$ & $22 \cdot 0,22 \cdot 6$ & 21.5 & $21 \cdot 2,21 \cdot 7$ & $20 \cdot 6$ & $20 \cdot 3,20 \cdot 8$ & $19 \cdot 4$ & $19 \cdot 1,19 \cdot 6$ & $17 \cdot 9$ & $17 \cdot 6,18 \cdot 2$ & -0.4 & 0.0 & 0.512 & $<0.001$ \\
\hline $\mathrm{Ca}(\mathrm{mg})$ & 871.2 & $857 \cdot 2,885 \cdot 3$ & $900 \cdot 3$ & $886 \cdot 2,914.5$ & 877.8 & $863.8,891.9$ & $846 \cdot 4$ & $832 \cdot 5,860 \cdot 4$ & $797 \cdot 8$ & $783.8,811.8$ & $-9 \cdot 1$ & 0.8 & 0.419 & $<0.001$ \\
\hline $\mathrm{Fe}(\mathrm{mg})$ & 11.5 & $11 \cdot 3,11 \cdot 6$ & $11 \cdot 3$ & $11 \cdot 1,11 \cdot 4$ & $11 \cdot 0$ & $10 \cdot 9,11 \cdot 2$ & $10 \cdot 8$ & $10 \cdot 7,11 \cdot 0$ & $10 \cdot 3$ & $10 \cdot 2,10.5$ & -0.1 & 0.0 & 0.619 & $<0.001$ \\
\hline Thiamin (mg) & 1.9 & $1 \cdot 9,2 \cdot 0$ & 1.9 & $1.9,1.9$ & $1 \cdot 8$ & $1 \cdot 8,1 \cdot 8$ & $1 \cdot 7$ & $1 \cdot 7,1 \cdot 8$ & 1.7 & $1 \cdot 6,1 \cdot 7$ & -0.0 & 0.0 & 0.312 & $<0.001$ \\
\hline Vitamin C (mg) & 115.5 & $111.6,119.4$ & 114.9 & $111.0,118.8$ & $110 \cdot 7$ & $106 \cdot 8,114.5$ & $102 \cdot 3$ & $98 \cdot 5,106 \cdot 2$ & $96 \cdot 7$ & $92 \cdot 8,100 \cdot 5$ & $-2 \cdot 1$ & 0.2 & 0.095 & $<0.001$ \\
\hline Vitamin A RE $(\mu \mathrm{g})$ & 793.7 & $777 \cdot 4,810 \cdot 0$ & $762 \cdot 1$ & $745 \cdot 7,778.5$ & $760 \cdot 7$ & $744.4,777 \cdot 0$ & $720 \cdot 2$ & $704 \cdot 0,736 \cdot 4$ & $683 \cdot 3$ & $667 \cdot 1,699 \cdot 6$ & -11.2 & 1.0 & 0.204 & $<0.001$ \\
\hline Riboflavin (mg) & $2 \cdot 6$ & $2 \cdot 5,2 \cdot 6$ & $2 \cdot 7$ & $2 \cdot 6,2 \cdot 7$ & $2 \cdot 6$ & $2 \cdot 5,2 \cdot 6$ & $2 \cdot 4$ & $2.4,2.5$ & $2 \cdot 4$ & $2 \cdot 3,2 \cdot 4$ & -0.0 & 0.0 & 0.294 & $<0.001$ \\
\hline DFE $(\mu \mathrm{g})$ & $508 \cdot 2$ & $496.4,519.9$ & $506 \cdot 3$ & $494.5,518 \cdot 2$ & $486 \cdot 7$ & $474.9,498.4$ & 448.9 & $437 \cdot 2,460 \cdot 6$ & 424.9 & $413 \cdot 2,436.6$ & -8.9 & 0.7 & 0.149 & $<0.001$ \\
\hline Vitamin D $(\mu \mathrm{g})$ & $3 \cdot 3$ & $3 \cdot 2,3 \cdot 3$ & $3 \cdot 3$ & $3 \cdot 2,3 \cdot 4$ & $3 \cdot 2$ & $3 \cdot 1,3 \cdot 3$ & 3.0 & $2 \cdot 9,3 \cdot 1$ & $2 \cdot 7$ & $2 \cdot 7,2 \cdot 8$ & -0.1 & 0.0 & 0.293 & $<0.001$ \\
\hline Vitamin $\mathrm{E}(\mathrm{mg})$ & $6 \cdot 1$ & $6 \cdot 0,6 \cdot 2$ & 5.9 & $5.8,5.9$ & $5 \cdot 8$ & $5 \cdot 7,5 \cdot 9$ & $5 \cdot 6$ & $5 \cdot 5,5 \cdot 7$ & $5 \cdot 2$ & $5 \cdot 1,5 \cdot 3$ & -0.1 & 0.0 & 0.519 & $<0.001$ \\
\hline$P(\mathrm{mg})$ & $1414 \cdot 8$ & $1403 \cdot 7,1425 \cdot 9$ & $1400 \cdot 6$ & $1389 \cdot 4,1411 \cdot 8$ & 1371.4 & $1360 \cdot 2,1382 \cdot 5$ & $1330 \cdot 4$ & $1319 \cdot 3,1341 \cdot 4$ & $1254 \cdot 5$ & $1243 \cdot 5,1265 \cdot 6$ & $-16 \cdot 7$ & 0.7 & 0.781 & $<0.001$ \\
\hline $\mathrm{Mg}(\mathrm{mg})$ & 297.2 & $294.6,299.9$ & $290 \cdot 7$ & $288 \cdot 1,293 \cdot 4$ & $281 \cdot 1$ & $278 \cdot 5,283 \cdot 8$ & 269.5 & $266 \cdot 9,272 \cdot 2$ & 254.2 & $251 \cdot 6,256 \cdot 9$ & -4.4 & 0.2 & 0.702 & $<0.001$ \\
\hline $\mathrm{Zn}(\mathrm{mg})$ & $10 \cdot 8$ & $10.7,10.9$ & $10 \cdot 5$ & $10 \cdot 3,10 \cdot 6$ & $10 \cdot 1$ & $10 \cdot 0,10 \cdot 2$ & 9.8 & $9.7,9.9$ & $9 \cdot 4$ & $9 \cdot 3,9.5$ & -0.1 & 0.0 & 0.693 & $<0.001$ \\
\hline $\mathrm{K}(\mathrm{mg})$ & $2827 \cdot 0$ & $2800 \cdot 4,2853 \cdot 5$ & $2753 \cdot 3$ & $2726 \cdot 6,2780 \cdot 1$ & $2700 \cdot 3$ & $2673 \cdot 7,2727 \cdot 0$ & $2614 \cdot 2$ & $2587 \cdot 7,2640 \cdot 6$ & $2471 \cdot 0$ & $2444 \cdot 6,2497.5$ & $-36 \cdot 1$ & 1.6 & 0.647 & $<0.001$ \\
\hline $\mathrm{I}(\mu \mathrm{g})$ & 134.2 & $131 \cdot 7,136 \cdot 8$ & $1345 \cdot 8$ & $133 \cdot 2,138 \cdot 3$ & 131.8 & $129 \cdot 2,134 \cdot 3$ & $128 \cdot 3$ & $125 \cdot 8,130 \cdot 8$ & $122 \cdot 0$ & $119 \cdot 5,124 \cdot 6$ & -1.4 & 0.2 & 0.346 & $<0.001$ \\
\hline $\mathrm{Na}(\mathrm{mg})$ & 2495.7 & $2469 \cdot 7,2521 \cdot 7$ & $2438 \cdot 3$ & $2412 \cdot 2,2464 \cdot 4$ & $2408 \cdot 7$ & $2382 \cdot 7,2434.7$ & 2398.5 & $2372 \cdot 6,2424 \cdot 3$ & 2337.9 & $2312 \cdot 0,2363 \cdot 8$ & $-12 \cdot 4$ & 1.6 & 0.704 & $<0.001$ \\
\hline
\end{tabular}

LC $n$-3 PUFA, long chain $n-3$ PUFA; RE, retinol equivalents; DFE, dietary folate equivalents.

"Values are estimated marginal means calculated using ANCOVA, with total energy intake and BMI $z$ scores as covariates except for energy, where only BMI z score was included as a covariate.

$\dagger$ Usual intake calculated using the multiple source method ${ }^{(27)}$. Because of the transformation, the sum of added sugars from core foods and extra foods is slightly different from total usual added sugar intake.

$\ddagger \beta$ and $P_{\text {trend }}$ calculated using linear regression with nutrient as the dependent variable, \%E from added sugars as a continuous independent variable and age, sex, total energy intake and $\mathrm{BMI} z$-scores as covariates.

$\S$ 'Core' and 'extra' foods as defined by Rangan et al. (30,31). $^{\circ}$ 
Table 2. Mean* intake $\dagger$ of energy and nutrient according to age- and sex-specific quintiles of percentage energy (\%E) from total sugars (Mean values and $95 \%$ confidence intervals; $\beta$ coefficients with their standard errors)

\begin{tabular}{|c|c|c|c|c|c|c|c|c|c|c|c|c|c|c|}
\hline \multirow[b]{3}{*}{ Nutrients } & \multicolumn{10}{|c|}{$\% \mathrm{E}$ from total sugars } & \multirow[b]{3}{*}{$\beta \ddagger$} & \multirow[b]{3}{*}{ SE } & \multirow[b]{3}{*}{$R^{2}$} & \multirow[b]{3}{*}{$P_{\text {trend }} \ddagger$} \\
\hline & \multicolumn{2}{|r|}{ Q1 } & \multicolumn{2}{|r|}{ Q2 } & \multicolumn{2}{|r|}{ Q3 } & \multicolumn{2}{|r|}{ Q4 } & \multicolumn{2}{|r|}{ Q5 } & & & & \\
\hline & Mean & $95 \% \mathrm{Cl}$ & Mean & $95 \% \mathrm{Cl}$ & Mean & $95 \% \mathrm{Cl}$ & Mean & $95 \% \mathrm{Cl}$ & Mean & $95 \% \mathrm{Cl}$ & & & & \\
\hline Total sugars $(\mathrm{g})$ & 90.6 & $90 \cdot 0,91 \cdot 3$ & 109.0 & $108 \cdot 3,109 \cdot 6$ & 120.3 & $119 \cdot 7,121 \cdot 0$ & 131.5 & $130 \cdot 8,132 \cdot 1$ & 147.8 & $147 \cdot 2,148.5$ & 5.0 & 0.0 & 0.967 & $<0.001$ \\
\hline$\%$ E total sugars & $19 \cdot 5$ & $19 \cdot 4,19 \cdot 6$ & 23.3 & $23 \cdot 2,23 \cdot 4$ & 25.5 & $25 \cdot 4,25 \cdot 6$ & $27 \cdot 7$ & $27 \cdot 6,27 \cdot 8$ & 31.2 & $31 \cdot 1,31 \cdot 3$ & - & - & _- & - \\
\hline Energy $(\mathrm{kJ})$ & 7989.8 & $7862 \cdot 0,8117 \cdot 7$ & 8141.6 & $8015 \cdot 0,8268 \cdot 1$ & 8204.6 & $8078.3,8330.9$ & 8176.9 & $8050 \cdot 2,8303 \cdot 7$ & $8026 \cdot 3$ & $7898 \cdot 9,8153 \cdot 7$ & -0.0 & 4.5 & 0.587 & 0.994 \\
\hline Protein $(\mathrm{g})$ & 84.3 & $83 \cdot 6,84.9$ & 81.3 & $80 \cdot 7,82 \cdot 0$ & 79.2 & $78.5,79.9$ & $78 \cdot 2$ & $77.5,78.9$ & 75.0 & $74 \cdot 3,75 \cdot 7$ & -0.8 & 0.0 & 0.787 & $<0.001$ \\
\hline$\%$ E protein & $17 \cdot 6$ & $17 \cdot 5,17 \cdot 7$ & $17 \cdot 1$ & $16 \cdot 9,17 \cdot 2$ & $16 \cdot 7$ & $16 \cdot 6,16 \cdot 8$ & 16.5 & $16 \cdot 4,16 \cdot 6$ & 15.9 & $15 \cdot 8,16 \cdot 0$ & -0.1 & 0.0 & 0.148 & $<0.001$ \\
\hline Total fat $(\mathrm{g})$ & 71.5 & $71 \cdot 0,72 \cdot 0$ & $69 \cdot 8$ & $69 \cdot 3,70 \cdot 2$ & 68.7 & $68 \cdot 3,69 \cdot 2$ & 66.4 & $65 \cdot 9,66 \cdot 8$ & 63.6 & $63 \cdot 1,64.0$ & -0.7 & 0.0 & 0.855 & $<0.001$ \\
\hline$\%$ E total fat & $32 \cdot 2$ & $32 \cdot 0,32 \cdot 4$ & 31.3 & $31.1,31.5$ & 30.9 & $30 \cdot 7,31 \cdot 1$ & 29.8 & $29.6,30.0$ & 28.6 & $28 \cdot 4,28 \cdot 8$ & -0.3 & 0.0 & 0.199 & $<0.001$ \\
\hline SFA (g) & 31.1 & $30 \cdot 8,31.4$ & $31 \cdot 1$ & $30 \cdot 8,31 \cdot 4$ & $31 \cdot 2$ & $0.9,31.5$ & 30.3 & $30 \cdot 0,30 \cdot 7$ & 29.5 & $29 \cdot 2,29 \cdot 9$ & -0.2 & 0.0 & 0.709 & $<0.001$ \\
\hline$\%$ E SFA & 14.0 & $13 \cdot 9,14 \cdot 1$ & 14.0 & $13 \cdot 8,14 \cdot 1$ & 14.0 & $13 \cdot 9,14 \cdot 2$ & 13.6 & $13 \cdot 5,13.8$ & 13.2 & $13 \cdot 1,13.4$ & -0.1 & 0.0 & 0.046 & $<0.001$ \\
\hline MUFA (g) & 25.4 & $25 \cdot 2,25 \cdot 6$ & 24.4 & $24 \cdot 2,24 \cdot 6$ & 23.9 & $23 \cdot 7,24 \cdot 0$ & 22.9 & $22 \cdot 7,23 \cdot 1$ & 21.7 & $21 \cdot 5,21 \cdot 9$ & -0.3 & 0.0 & 0.827 & $<0 \cdots 001$ \\
\hline PUFA (g) & 9.6 & $9 \cdot 4,9.7$ & 9.1 & $9 \cdot 0,9 \cdot 2$ & 8.7 & $8.6,8.8$ & 8.3 & $8 \cdot 2,8 \cdot 4$ & 7.8 & $7.7,7.9$ & -0.2 & 0.0 & 0.653 & $<0.001$ \\
\hline LC $n-3$ PUFA (mg) & $134 \cdot 7$ & $131 \cdot 2,138.3$ & 120.5 & $117 \cdot 0,124 \cdot 0$ & 114.3 & $110.8,117.8$ & $110 \cdot 1$ & $106 \cdot 6,113.6$ & 103.7 & $100 \cdot 2,107 \cdot 3$ & $-2 \cdot 8$ & 0.2 & 0.176 & $<0.001$ \\
\hline Total carbohydrate (g) & $229 \cdot 8$ & $228 \cdot 6,231 \cdot 0$ & 238.2 & $237 \cdot 0,239 \cdot 4$ & 243.8 & $242 \cdot 6,252 \cdot 3$ & $251 \cdot 1$ & $249 \cdot 9,252 \cdot 3$ & $261 \cdot 7$ & $260.5,262 \cdot 9$ & $2 \cdot 8$ & 0.1 & 0.909 & $<0.001$ \\
\hline$\% \mathrm{E}$ carbohydrates & 49.0 & $48 \cdot 8,49 \cdot 2$ & 50.5 & $50 \cdot 3,50 \cdot 7$ & 51.5 & $51 \cdot 2,51 \cdot 7$ & $52 \cdot 8$ & $52 \cdot 6,53 \cdot 0$ & 54.8 & $54 \cdot 6,55 \cdot 1$ & 0.5 & 0.0 & 0.358 & $<0.001$ \\
\hline Added sugars (g) & 43.1 & $42 \cdot 1,44 \cdot 1$ & $51 \cdot 0$ & $50 \cdot 0,52 \cdot 0$ & 57.7 & $56 \cdot 8,58 \cdot 7$ & $62 \cdot 3$ & $61 \cdot 3,63 \cdot 3$ & $73 \cdot 1$ & $72 \cdot 1,74 \cdot 1$ & 2.6 & 0.1 & 0.662 & $<0.001$ \\
\hline From core foods $\S$ & 9.4 & $8.9,9.8$ & $10 \cdot 7$ & $10 \cdot 3,11 \cdot 2$ & $11 \cdot 7$ & $11 \cdot 3,12 \cdot 1$ & $12 \cdot 9$ & $12 \cdot 5,13 \cdot 3$ & 13.3 & $12 \cdot 9,13 \cdot 7$ & 0.4 & 0.0 & 0.120 & $<0.001$ \\
\hline From extra foods§ & 34.1 & $33 \cdot 0,35 \cdot 2$ & $40 \cdot 6$ & $39.6,41 \cdot 7$ & $46 \cdot 1$ & $45 \cdot 1,47 \cdot 2$ & $49 \cdot 8$ & $48.7,50 \cdot 8$ & $59 \cdot 7$ & $58 \cdot 7,60 \cdot 8$ & $2 \cdot 2$ & 0.1 & 0.570 & $<0.001$ \\
\hline$\%$ E added sugars & 8.6 & $8 \cdot 4,8 \cdot 8$ & $10 \cdot 4$ & $10 \cdot 2,10 \cdot 6$ & 11.8 & $11 \cdot 6,12 \cdot 0$ & $12 \cdot 8$ & $12 \cdot 6,13 \cdot 0$ & $15 \cdot 2$ & $14 \cdot 9,15 \cdot 4$ & 0.6 & 0.0 & 0.438 & $<0.001$ \\
\hline From core foods§ & 2.0 & $1 \cdot 9,2 \cdot 1$ & 2.3 & $2 \cdot 2,2 \cdot 4$ & 2.5 & $2 \cdot 4,2 \cdot 6$ & $2 \cdot 7$ & $2 \cdot 7,2 \cdot 8$ & 2.9 & $2 \cdot 8,2 \cdot 9$ & 0.1 & 0.0 & 0.093 & $<0.001$ \\
\hline From extra foods§ & $6 \cdot 7$ & $6 \cdot 5,7 \cdot 0$ & $8 \cdot 2$ & $8 \cdot 0,8 \cdot 4$ & $9 \cdot 3$ & $9.1,9.5$ & $10 \cdot 1$ & $9 \cdot 8,10 \cdot 3$ & $12 \cdot 2$ & $12 \cdot 0,12 \cdot 4$ & 0.5 & 0.0 & 0.356 & $<0.001$ \\
\hline Starch $(g)$ & $135 \cdot 2$ & $134 \cdot 1,136 \cdot 3$ & $127 \cdot 0$ & $125 \cdot 8,128 \cdot 1$ & 121.8 & $120.6,122.9$ & $118 \cdot 1$ & $117 \cdot 0,119.2$ & $112 \cdot 3$ & $111.2,113.4$ & -2.0 & 0.1 & 0.778 & $<0.001$ \\
\hline$\%$ E starch & 28.5 & $28 \cdot 3,28 \cdot 7$ & $26 \cdot 7$ & $26 \cdot 5,26 \cdot 9$ & $25 \cdot 5$ & $25 \cdot 3,25 \cdot 8$ & 24.8 & $24 \cdot 6,25 \cdot 1$ & 23.5 & $23 \cdot 3,23 \cdot 8$ & -0.4 & 0.0 & 0.255 & $<0.001$ \\
\hline Fibre (g) & 20.4 & $20 \cdot 1,20 \cdot 7$ & $20 \cdot 7$ & $20 \cdot 4,21 \cdot 0$ & $20 \cdot 3$ & $20 \cdot 0,20 \cdot 6$ & 20.4 & $20 \cdot 1,20 \cdot 7$ & $19 \cdot 8$ & $19 \cdot 5,20 \cdot 0$ & -0.0 & 0.0 & 0.433 & 0.003 \\
\hline $\mathrm{Ca}(\mathrm{mg})$ & 781.9 & $767 \cdot 8,796 \cdot 0$ & $855 \cdot 1$ & $841 \cdot 2,869 \cdot 0$ & 869.8 & $855.9,883.7$ & $896 \cdot 3$ & $882 \cdot 4,910 \cdot 3$ & 888.5 & $874.5,902.5$ & $8 \cdot 3$ & 0.7 & 0.420 & $<0.001$ \\
\hline $\mathrm{Fe}(\mathrm{mg})$ & $11 \cdot 1$ & $10 \cdot 9,11 \cdot 2$ & 11.1 & $11 \cdot 0,11 \cdot 3$ & 11.0 & $10 \cdot 8,11 \cdot 1$ & 11.1 & $11 \cdot 0,11 \cdot 3$ & 10.6 & $10 \cdot 5,10 \cdot 7$ & -0.0 & 0.0 & 0.607 & $<0.001$ \\
\hline Thiamin (mg) & 1.81 & $1.78,1.85$ & 1.84 & $1.80,1.88$ & 1.81 & $1.77,1.84$ & 1.81 & $1.78,1.85$ & 1.72 & $1.68,1.76$ & -0.0 & 0.0 & 0.288 & $<0.001$ \\
\hline Vitamin C (mg) & 86.9 & $83 \cdot 1,90 \cdot 7$ & $102 \cdot 8$ & $99.0,106.6$ & $105 \cdot 5$ & $101.7,109.3$ & $118 \cdot 4$ & $114 \cdot 6,122 \cdot 2$ & $126 \cdot 2$ & $122 \cdot 4,130.0$ & 3.8 & 0.2 & 0.142 & $<0.001$ \\
\hline Vitamin A RE ( $\mu \mathrm{g})$ & $697 \cdot 7$ & $681 \cdot 1,714.2$ & $750 \cdot 2$ & $733.8,766.6$ & 755.4 & $739.1,771 \cdot 7$ & 764.2 & $747 \cdot 8,780 \cdot 6$ & 750.9 & $734.4,767 \cdot 4$ & 4.5 & 0.9 & 0.184 & $<0.001$ \\
\hline Riboflavin (mg) & 2.29 & $2 \cdot 24,2 \cdot 35$ & 2.53 & $2 \cdot 48,2.58$ & 2.56 & $2 \cdot 51,2 \cdot 61$ & 2.63 & $2.58,2.68$ & 2.57 & $2 \cdot 52,2.63$ & 0.0 & 0.0 & 0.291 & $<0.001$ \\
\hline DFE $(\mu \mathrm{g})$ & 437.9 & $426.0,449.9$ & $475 \cdot 7$ & $463.8,487.5$ & 480.5 & $468 \cdot 6,492 \cdot 3$ & 499.0 & $487.2,510.9$ & $480 \cdot 2$ & $468 \cdot 3,492 \cdot 2$ & 3.9 & 0.7 & 0.125 & $<0.001$ \\
\hline Vitamin D $(\mu \mathrm{g})$ & 3.00 & $2.93,3.07$ & $3 \cdot 16$ & $3.09,3.24$ & 3.15 & $3.08,3.22$ & $3 \cdot 15$ & $3.08,3.22$ & 2.99 & $2.91,3.06$ & -0.0 & 0.0 & 0.256 & 0.132 \\
\hline Vitamin $\mathrm{E}(\mathrm{mg})$ & $6 \cdot 13$ & $6 \cdot 05,6 \cdot 22$ & $5 \cdot 87$ & $5.79,5.95$ & 5.64 & $5 \cdot 55,5 \cdot 72$ & $5 \cdot 63$ & $5 \cdot 55,5 \cdot 71$ & $5 \cdot 26$ & $5 \cdot 18,5 \cdot 34$ & -0.1 & 0.0 & 0.513 & $<0.001$ \\
\hline$P(m g)$ & $1358 \cdot 3$ & $1346 \cdot 5,1370 \cdot 1$ & $1378 \cdot 7$ & $1367 \cdot 1,1390 \cdot 4$ & $1350 \cdot 1$ & $1348 \cdot 4,1371 \cdot 7$ & 1354.5 & $1342 \cdot 9,1366 \cdot 2$ & $1318 \cdot 0$ & $1306 \cdot 3,1329 \cdot 7$ & $-4 \cdot 4$ & 0.6 & 0.751 & $<0.001$ \\
\hline $\mathrm{Mg}(\mathrm{mg})$ & 277.0 & $274 \cdot 1,279 \cdot 8$ & $282 \cdot 2$ & $279 \cdot 4,285 \cdot 0$ & 277.5 & $274.7,280 \cdot 4$ & 281.4 & $278 \cdot 6,284 \cdot 3$ & 274.3 & $271 \cdot 4,277 \cdot 1$ & -0.2 & 0.2 & 0.649 & 0.325 \\
\hline $\mathrm{Zn}(\mathrm{mg})$ & 10.5 & $10 \cdot 4,10 \cdot 6$ & 10.4 & $10 \cdot 2,10.5$ & 10.1 & $10 \cdot 0,10 \cdot 2$ & 9.9 & $9.8,10 \cdot 0$ & 9.7 & $9.6,9.8$ & -0.1 & 0.0 & 0.672 & $<0.001$ \\
\hline $\mathrm{K}(\mathrm{mg})$ & $2542 \cdot 6$ & $2515 \cdot 1,2570 \cdot 1$ & $2640 \cdot 2$ & $2613 \cdot 0,2667 \cdot 5$ & $2675 \cdot 1$ & $2647 \cdot 9,2702 \cdot 2$ & $2757 \cdot 6$ & $2730 \cdot 3,2784 \cdot 8$ & $2746 \cdot 3$ & $2718 \cdot 9,2773 \cdot 8$ & $19 \cdot 3$ & 1.5 & 0.620 & $<0.001$ \\
\hline I $(\mu \mathrm{g})$ & $115 \cdot 7$ & $113 \cdot 2,118 \cdot 2$ & $130 \cdot 2$ & $127 \cdot 7,132 \cdot 7$ & 130.1 & $127 \cdot 6,132 \cdot 6$ & 136.5 & $134.0,139.0$ & 139.4 & $136 \cdot 9,141 \cdot 9$ & 1.8 & 0.1 & 0.361 & $<0.001$ \\
\hline $\mathrm{Na}(\mathrm{mg})$ & 2581.0 & $2555 \cdot 6,2606 \cdot 4$ & 2461.8 & $2436 \cdot 7,2487 \cdot 0$ & $2415 \cdot 8$ & $2390.7,2440.9$ & 2350.7 & $2325 \cdot 5,2375 \cdot 9$ & 2269.5 & $2244.2,2294.9$ & $-27 \cdot 1$ & 1.3 & 0.726 & $<0.001$ \\
\hline
\end{tabular}

$\%$ E, percentage energy; LC $n-3$ PUFA, long-chain $n$-3 PUFA; RE, retinol equivalents; DFE, dietary folate equivalents.

* Values are estimated marginal means calculated using ANCOVA, with total energy intake and BMI $z$-scores as covariates except for energy, where only BMI z-score was included as a covariate.

† Usual intake calculated using the multiple source method ${ }^{(2 \pi)}$. Because of the transformation, the sum of added sugars from core foods and extra foods is slightly different from total usual added sugar intake.

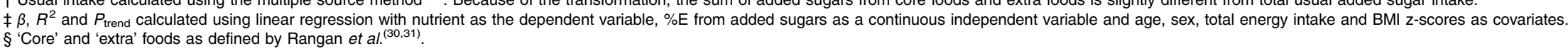


Table 3. Percentage of subjects not meeting nutrient reference values (NRV) for Australia and New Zealand* according to age- and sex-specific quintiles $(\mathrm{Q})$ of percentage energy $(\% \mathrm{E})$ from added sugars, stratified by source (Odds ratios and $95 \%$ confidence intervals)

\begin{tabular}{|c|c|c|c|c|c|c|c|c|c|c|c|c|}
\hline \multirow[b]{2}{*}{ Nutrients } & \multirow[b]{2}{*}{ Source of added sugars $†$} & \multicolumn{5}{|c|}{$\% \mathrm{E}$ from added sugars } & \multirow[b]{2}{*}{$\chi^{2}$} & \multirow[b]{2}{*}{$P \ddagger$} & \multirow[b]{2}{*}{ OR§ } & \multirow[b]{2}{*}{$95 \% \mathrm{Cl}$} & \multirow[b]{2}{*}{$R^{2}$} & \multirow[b]{2}{*}{$P_{\text {trend }} \S$} \\
\hline & & Q1 & Q2 & Q3 & Q4 & Q5 & & & & & & \\
\hline \multirow[t]{3}{*}{ SFA } & All foods & $94 \cdot 3$ & $96 \cdot 3$ & $96 \cdot 9$ & 97.5 & $96 \cdot 6$ & $13 \cdot 4$ & 0.010 & 1.04 & $1.00,1.09$ & 0.039 & 0.065 \\
\hline & From core foods only & 96.5 & 97.6 & $96 \cdot 7$ & $96 \cdot 5$ & 94.2 & $15 \cdot 0$ & 0.005 & 0.83 & $0.74,0.92$ & 0.045 & $<0.001$ \\
\hline & From extra foods only & 93.5 & 96.5 & $96 \cdot 3$ & $98 \cdot 6$ & $96 \cdot 6$ & $30 \cdot 6$ & $<0.001$ & $1 \cdot 10$ & $1.04,1 \cdot 15$ & 0.047 & $<0.001$ \\
\hline \multirow[t]{3}{*}{ LC $n$-3 PUFA } & All foods & 7.9 & 8.8 & 6.5 & 8.6 & $10 \cdot 7$ & $9 \cdot 7$ & 0.045 & 1.04 & $1.01,1.07$ & 0.223 & 0.016 \\
\hline & From core foods only & 8.5 & $7 \cdot 8$ & 8.0 & 8.2 & $10 \cdot 1$ & 3.5 & 0.474 & $1 \cdot 10$ & $1.00,1 \cdot 20$ & 0.222 & 0.043 \\
\hline & From extra foods only & 8.9 & $8 \cdot 2$ & 7.5 & $8 \cdot 4$ & 9.5 & $2 \cdot 4$ & 0.656 & 1.02 & $0.99,1.05$ & 0.220 & 0.248 \\
\hline \multirow{3}{*}{ Fibre } & All foods & 38.2 & 42.5 & $46 \cdot 7$ & 55.4 & $66 \cdot 7$ & $170 \cdot 2$ & $<0.001$ & 1.17 & $1 \cdot 15,1 \cdot 19$ & 0.239 & $<0.001$ \\
\hline & From core foods only & $54 \cdot 3$ & $47 \cdot 0$ & $47 \cdot 3$ & $47 \cdot 6$ & $53 \cdot 6$ & $17 \cdot 7$ & 0.001 & 1.05 & $0.99,1.10$ & 0.164 & 0.090 \\
\hline & From extra foods only & 38.7 & $45 \cdot 6$ & $45 \cdot 8$ & $52 \cdot 9$ & $66 \cdot 7$ & $149 \cdot 7$ & $<0.001$ & $1 \cdot 17$ & $1 \cdot 15,1 \cdot 19$ & 0.235 & $<0.001$ \\
\hline \multirow[t]{3}{*}{$\mathrm{Ca}$} & All foods & 35.1 & 26.5 & 29.9 & $32 \cdot 0$ & $40 \cdot 7$ & 43.7 & $<0.001$ & $1 \cdot 10$ & $1.07,1.13$ & 0.587 & $<0.001$ \\
\hline & From core foods only & 41.7 & $36 \cdot 1$ & 32.9 & $26 \cdot 6$ & 26.9 & $61 \cdot 7$ & $<0.001$ & 0.71 & $0.65,0.77$ & 0.590 & $<0.001$ \\
\hline & From extra foods only & 30.8 & $28 \cdot 8$ & $28 \cdot 0$ & 32.9 & 43.6 & $59 \cdot 7$ & $<0.001$ & 1.15 & $1 \cdot 12,1 \cdot 18$ & 0.599 & $<0.001$ \\
\hline \multirow[t]{3}{*}{$\mathrm{Fe}$} & All foods & 0.2 & 0.8 & 0.7 & 0.8 & $1 \cdot 3$ & $6 \cdot 3$ & 0.175 & 1.24 & $1 \cdot 13,1.37$ & 0.527 & $<0.001$ \\
\hline & From core foods only & 1.3 & 0.6 & 0.6 & 0.6 & 0.9 & $4 \cdot 2$ & 0.381 & 1.00 & $0.74,1.36$ & 0.467 & 0.986 \\
\hline & From extra foods only & 0.5 & 0.2 & 0.8 & $1 \cdot 1$ & 1.2 & $7 \cdot 1$ & 0.128 & 1.20 & $1 \cdot 10,1.31$ & 0.508 & $<0.001$ \\
\hline \multirow[t]{3}{*}{ Thiamin } & All foods & 0.1 & 0.2 & 0.2 & 0.5 & 0.8 & $7 \cdot 2$ & 0.125 & 1.28 & $1.15,1.42$ & 0.380 & $<0.001$ \\
\hline & From core foods only & 0.8 & 0.4 & 0.0 & 0.7 & 0.1 & $10 \cdot 9$ & 0.027 & 0.79 & $0.50,1.27$ & 0.284 & 0.336 \\
\hline & From extra foods only & 0.1 & 0.2 & 0.1 & 0.5 & $1 \cdot 0$ & 11.0 & 0.026 & 1.27 & $1 \cdot 14,1.42$ & 0.365 & $<0.001$ \\
\hline Vitamin C & All foods & 0.7 & 0.2 & 0.2 & 0.0 & 0.2 & $8 \cdot 0$ & 0.090 & 0.88 & $0.75,1.03$ & 0.118 & 0.103 \\
\hline & From core foods only & $1 \cdot 1$ & 0.0 & 0.2 & 0.2 & 0.0 & 21.4 & $<0.001$ & 0.36 & $0.17,0.79$ & 0.156 & 0.011 \\
\hline & From extra foods only & 0.5 & 0.4 & 0.2 & 0.2 & 0.2 & 1.2 & 0.872 & 0.97 & $0.84,1.12$ & 0.103 & 0.647 \\
\hline Vitamin A RE & All foods & $6 \cdot 0$ & 5.5 & 4.6 & $6 \cdot 1$ & $10 \cdot 9$ & $32 \cdot 3$ & $<0.001$ & 1.11 & $1.07,1.15$ & 0.286 & $<0.001$ \\
\hline & From core foods only & 9.1 & $7 \cdot 2$ & $5 \cdot 1$ & $4 \cdot 1$ & 7.7 & $21 \cdot 7$ & $<0.001$ & 0.94 & $0.84,1.05$ & 0.265 & 0.246 \\
\hline & From extra foods only & $6 \cdot 7$ & $4 \cdot 8$ & $5 \cdot 2$ & $5 \cdot 3$ & $11 \cdot 2$ & $37 \cdot 1$ & $<0.001$ & $1 \cdot 11$ & $1.07,1.15$ & 0.286 & $<0.001$ \\
\hline Riboflavin & All foods & 0.0 & 0.0 & 0.5 & 0.0 & 0.5 & $12 \cdot 1$ & 0.017 & 1.37 & $1 \cdot 16,1 \cdot 62$ & 0.526 & $<0.001$ \\
\hline & From core foods only & 0.4 & 0.2 & 0.1 & 0.0 & 0.2 & 3.3 & 0.513 & 0.58 & $0.26,1.29$ & 0.419 & 0.179 \\
\hline & From extra foods only & 0.0 & 0.0 & 0.4 & $0 \cdot 1$ & 0.5 & $8 \cdot 3$ & 0.081 & 1.46 & $1.20,1.78$ & 0.554 & $<0.001$ \\
\hline DFE & All foods & 3.1 & 2.9 & 3.9 & $5 \cdot 3$ & 9.0 & 44.8 & $<0.001$ & $1 \cdot 18$ & $1.14,1.23$ & 0.370 & $<0.001$ \\
\hline & From core foods only & 8.3 & $5 \cdot 2$ & 4.4 & 3.5 & $2 \cdot 8$ & 33.5 & $<0.001$ & 0.68 & $0.59,0.80$ & 0.340 & $<0.001$ \\
\hline & From extra foods only & $2 \cdot 8$ & $2 \cdot 4$ & 4.0 & $5 \cdot 7$ & $9 \cdot 4$ & $57 \cdot 4$ & $<0.001$ & 1.21 & $1 \cdot 16,1 \cdot 26$ & 0.385 & $<0.001$ \\
\hline Vitamin D & All foods & $90 \cdot 9$ & $91 \cdot 9$ & 91.8 & $95 \cdot 5$ & $97 \cdot 6$ & 44.9 & $<0.001$ & $1 \cdot 20$ & $1 \cdot 15,1 \cdot 25$ & 0.262 & $<0.001$ \\
\hline & From core foods only & 93.3 & 93.4 & $93 \cdot 1$ & 93.9 & 93.9 & 0.7 & 0.951 & 1.03 & $0.92,1.15$ & 0.216 & 0.645 \\
\hline & From extra foods only & $90 \cdot 3$ & 91.0 & 93.6 & $95 \cdot 3$ & $97 \cdot 4$ & $48 \cdot 3$ & $<0.001$ & 1.20 & $1 \cdot 15,1 \cdot 26$ & 0.263 & $<0.001$ \\
\hline Vitamin $\mathrm{E}$ & All foods & $77 \cdot 3$ & $82 \cdot 3$ & 81.5 & $87 \cdot 1$ & 91.0 & $69 \cdot 1$ & $<0.001$ & 1.15 & $1 \cdot 12,1 \cdot 18$ & 0.180 & $<0.001$ \\
\hline & From core foods only & 83.4 & $81 \cdot 9$ & 82.5 & $85 \cdot 2$ & $86 \cdot 3$ & $8 \cdot 3$ & 0.083 & $1 \cdot 10$ & $1.03,1.18$ & 0.139 & 0.006 \\
\hline & From extra foods only & $77 \cdot 8$ & $81 \cdot 1$ & $85 \cdot 7$ & $84 \cdot 0$ & $90 \cdot 8$ & $58 \cdot 7$ & $<0.001$ & $1 \cdot 15$ & $1 \cdot 12,1 \cdot 18$ & 0.175 & $<0.001$ \\
\hline$P$ & All foods & 4.4 & 3.0 & 2.4 & 3.3 & $5 \cdot 6$ & 14.5 & 0.006 & 1.12 & $1.07,1.18$ & 0.425 & $<0.001$ \\
\hline & From core foods only & 5.4 & 3.6 & 3.3 & $3 \cdot 7$ & $2 \cdot 7$ & 9.8 & 0.044 & 0.81 & $0.69,0.95$ & 0.412 & 0.010 \\
\hline & From extra foods only & 3.1 & 4.1 & 3.0 & $2 \cdot 7$ & 5.7 & $14 \cdot 0$ & 0.007 & 1.13 & $1.08,1.18$ & 0.427 & $<0.001$ \\
\hline Mg & All foods & 7.9 & $8 \cdot 7$ & $8 \cdot 7$ & $10 \cdot 5$ & $16 \cdot 4$ & $42 \cdot 6$ & $<0.001$ & 1.19 & $1 \cdot 15,1 \cdot 24$ & 0.618 & $<0.001$ \\
\hline & From core foods only & 11.9 & 9.9 & $10 \cdot 8$ & 9.5 & $10 \cdot 1$ & $3 \cdot 1$ & 0.546 & 0.88 & $0.79,0.99$ & 0.586 & 0.030 \\
\hline & From extra foods only & 7.5 & 8.9 & 8.7 & $10 \cdot 6$ & $16 \cdot 3$ & $42 \cdot 7$ & $<0.001$ & 1.20 & $1 \cdot 16,1 \cdot 25$ & 0.622 & $<0.001$ \\
\hline $\mathrm{Zn}$ & All foods & 0.6 & 0.7 & $1 \cdot 2$ & 0.8 & $2 \cdot 4$ & $15 \cdot 9$ & 0.003 & 1.17 & $1.08,1.26$ & 0.535 & $<0.001$ \\
\hline & From core foods only & 0.6 & 1.4 & 1.1 & 1.6 & $1 \cdot 1$ & $4 \cdot 1$ & 0.398 & 1.05 & $0.82,1.34$ & 0.504 & 0.716 \\
\hline & From extra foods only & 1.0 & 0.5 & 0.6 & 1.4 & $2 \cdot 2$ & $14 \cdot 2$ & 0.007 & 1.14 & $1.05,1.23$ & 0.525 & $<0.001$ \\
\hline $\mathrm{K}$ & All foods & $37 \cdot 0$ & $36 \cdot 4$ & $35 \cdot 8$ & $44 \cdot 2$ & $55 \cdot 2$ & $92 \cdot 9$ & $<0.001$ & $1 \cdot 14$ & $1 \cdot 11,1 \cdot 16$ & 0.302 & $<0.001$ \\
\hline & From core foods only & $50 \cdot 3$ & $41 \cdot 3$ & $40 \cdot 0$ & 38.6 & $38 \cdot 3$ & 33.5 & $<0.001$ & 0.89 & $0.84,0.93$ & 0.263 & $<0.001$ \\
\hline & From extra foods only & 34.5 & $36 \cdot 6$ & 39.0 & $41 \cdot 0$ & $57 \cdot 4$ & 113.0 & $<0.001$ & $1 \cdot 16$ & $1 \cdot 14,1 \cdot 19$ & 0.317 & $<0.001$ \\
\hline I & All foods & 8.5 & 5.7 & 6.5 & 5.9 & 9.8 & $16 \cdot 0$ & 0.003 & 1.05 & $1.02,1.08$ & 0.221 & 0.004 \\
\hline & From core foc & $12 \cdot 2$ & 7.6 & 6.5 & $5 \cdot 2$ & $5 \cdot 0$ & $42 \cdot 5$ & $<0.001$ & 0.71 & $0.64,0.80$ & 0.236 & $<0.001$ \\
\hline & From extra foods only & $7 \cdot 1$ & $6 \cdot 2$ & $5 \cdot 8$ & $6 \cdot 6$ & $10 \cdot 8$ & $20 \cdot 1$ & $<0.001$ & 1.08 & $1.04,1.11$ & 0.228 & $<0.001$ \\
\hline $\mathrm{Na}$ & All foods & $94 \cdot 0$ & $93 \cdot 4$ & 94.8 & 94.5 & 94.4 & 1.8 & 0.773 & 0.97 & $0.93,1.00$ & 0.312 & 0.082 \\
\hline & From core foods only & $92 \cdot 6$ & $96 \cdot 3$ & $94 \cdot 0$ & $94 \cdot 7$ & $93 \cdot 3$ & 11.9 & 0.018 & 1.01 & $0.90,1.14$ & 0.310 & 0.823 \\
\hline & From extra foods only & 93.8 & 93.6 & 94.8 & $94 \cdot 2$ & 94.5 & 1.5 & 0.830 & 0.97 & $0.93,1.01$ & 0.312 & 0.090 \\
\hline
\end{tabular}

LC $n$-3 PUFA, long-chain $n$-3 PUFA; RE, retinol equivalents; DFE, dietary folate equivalents.

* For $\mathrm{Ca}, \mathrm{Fe}, \mathrm{I}, \mathrm{Zn}, \mathrm{Mg}, \mathrm{P}$, vitamin A RE, thiamin, riboflavin, DFE and vitamin C, intakes lower than the estimated average requirement were considered as not meeting the NRV; for K, LC $n$-3 PUFA, dietary fibre, vitamin D and vitamin E, intakes lower than the adequate intake were considered as not meeting the NRV; for Na, intakes higher than the upper level were considered as not meeting the NRV; for SFA, \%E $>10 \%$ was considered as not meeting the NRV.

† 'Core' and 'extra' foods as defined by Rangan et al. ${ }^{(30,31)}$

$\ddagger P$ value tested using Pearson's $\chi^{2}$ test.

$\S$ Odds ratios and $P_{\text {trend }}$ calculated using logistic regression with \%E from added sugars as a continuous independent variable and age, sex, total energy intake and BMI $z$-scores as covariates. 
Table 4. Percentage of subjects not meeting nutrient reference values (NRV) for Australia and New Zealand ${ }^{\star}$ according to age- and sex-specific quintiles (Q) of percentage energy (\%E) from total sugars

(Odds ratios and $95 \%$ confidence intervals)

\begin{tabular}{|c|c|c|c|c|c|c|c|c|c|c|c|}
\hline \multirow[b]{2}{*}{ Nutrients } & \multicolumn{5}{|c|}{$\% \mathrm{E}$ from total sugars } & \multirow[b]{2}{*}{$\chi^{2}$} & \multirow[b]{2}{*}{$P+$} & \multirow[b]{2}{*}{ OR $\ddagger$} & \multirow[b]{2}{*}{$95 \% \mathrm{Cl}$} & \multirow[b]{2}{*}{$R^{2}$} & \multirow[b]{2}{*}{$P_{\text {trend }} \ddagger$} \\
\hline & Q1 & Q2 & Q3 & Q4 & Q5 & & & & & & \\
\hline SFA & 96.0 & $96 \cdot 3$ & $97 \cdot 7$ & 96.9 & 94.8 & 11.0 & 0.027 & 0.98 & $0.94,1.02$ & 0.036 & 0.282 \\
\hline LC $n$-3 PUFA & $5 \cdot 8$ & 8.6 & 6.0 & 9.5 & $12 \cdot 6$ & 33.3 & $<0.001$ & 1.07 & $1.04,1.10$ & 0.232 & $<0.001$ \\
\hline Fibre & 51.4 & $49 \cdot 8$ & $46 \cdot 9$ & $47 \cdot 1$ & $54 \cdot 6$ & $13 \cdot 7$ & 0.008 & 1.00 & $0.98,1.01$ & 0.122 & 0.541 \\
\hline $\mathrm{Ca}$ & 41.3 & 35.3 & 29.0 & $27 \cdot 3$ & 31.2 & 47.5 & $<0.001$ & 0.94 & $0.92,0.96$ & 0.418 & $<0.001$ \\
\hline $\mathrm{Fe}$ & 0.7 & 0.5 & 0.5 & 0.6 & 1.6 & 9.2 & 0.057 & $1 \cdot 15$ & $1.06,1.25$ & 0.497 & $<0.001$ \\
\hline Thiamin & 0.5 & 0.2 & 0.2 & 0.4 & 0.7 & 3.4 & 0.501 & 1.13 & $1.01,1.25$ & 0.301 & 0.027 \\
\hline Vitamin C & 1.1 & 0.1 & 0.2 & 0.1 & 0.0 & $20 \cdot 6$ & $<0.001$ & 0.73 & $0.63,0.84$ & 0.218 & $<0.001$ \\
\hline Vitamin A RE & 9.7 & 5.4 & 5.9 & 4.6 & 7.5 & 21.9 & $<0.001$ & 0.96 & $0.94,1.00$ & 0.268 & 0.022 \\
\hline Riboflavin & 0.2 & 0.0 & 0.2 & 0.2 & 0.2 & $2 \cdot 0$ & 0.734 & $1 \cdot 13$ & $0.97,1.31$ & 0.420 & $0 \cdot 121$ \\
\hline DFE & 8.0 & 3.4 & 3.9 & 4.7 & 4.3 & $24 \cdot 0$ & $<0.001$ & 0.95 & $0.92,0.99$ & 0.105 & 0.011 \\
\hline Vitamin D & 94.3 & $92 \cdot 0$ & 93.1 & $93 \cdot 0$ & $95 \cdot 1$ & 8.0 & 0.092 & 1.03 & $0.99,1.06$ & 0.217 & 0.104 \\
\hline Vitamin E & $76 \cdot 1$ & 79.7 & $86 \cdot 3$ & 84.9 & $92 \cdot 3$ & $95 \cdot 7$ & $<0.001$ & $1 \cdot 12$ & $1.09,1.14$ & 0.171 & $<0.001$ \\
\hline $\mathrm{P}$ & 4.7 & 3.5 & $2 \cdot 8$ & 3.4 & 4.4 & $5 \cdot 7$ & 0.224 & 1.02 & $0.98,1.06$ & 0.407 & 0.419 \\
\hline $\mathrm{Mg}$ & $10 \cdot 9$ & $9 \cdot 4$ & $9 \cdot 3$ & $10 \cdot 0$ & $12 \cdot 6$ & 6.7 & 0.152 & 1.02 & $0.99,1.05$ & 0.585 & 0.266 \\
\hline $\mathrm{Zn}$ & 1.2 & 0.5 & 0.8 & 1.3 & 1.8 & 7.5 & 0.110 & 1.09 & $1.01,1.18$ & 0.513 & 0.028 \\
\hline $\mathrm{K}$ & $54 \cdot 3$ & $42 \cdot 0$ & 41.0 & 33.0 & 38.3 & 84.2 & $<0.001$ & 0.92 & $0.90,0.93$ & 0.285 & $<0.001$ \\
\hline 1 & 11.7 & 6.5 & 7.4 & 6.1 & 4.7 & 34.3 & $<0.001$ & 0.93 & $0.90,0.95$ & 0.231 & $<0.001$ \\
\hline $\mathrm{Na}$ & $96 \cdot 7$ & 94.0 & 94.8 & 93.4 & $92 \cdot 1$ & $18 \cdot 0$ & $<0.001$ & 0.92 & $0.89,0.95$ & 0.326 & $<0.001$ \\
\hline
\end{tabular}

LC $n$-3 PUFA, long-chain $n$-3 PUFA; RE, retinol equivalents; DFE, dietary folate equivalents.

* For $\mathrm{Ca}, \mathrm{Fe}, \mathrm{I}, \mathrm{Zn}, \mathrm{Mg}, \mathrm{P}$, vitamin A RE, thiamin, riboflavin, DFE and vitamin C, intakes lower than the estimated average requirement were considered as not meeting the NRV; for K, LC $n$-3 PUFA, dietary fibre, vitamin D and vitamin E, intakes lower than the adequate intake were considered as not meeting the NRV; for Na, intakes higher than the upper level were considered as not meeting the NRV; for SFA, \%E $>10 \%$ was considered as not meeting the NRV.

$\dagger P$ value tested using Pearson's $\chi^{2}$ test.

$\ddagger$ Odds ratios and $P_{\text {trend }}$ calculated using logistic regression with \%E from total sugars as a continuous independent variable and age, sex, total energy intake and BMI $z$-scores as covariates.

The magnitude of the associations observed in our study, although statistically significant, was small. This likely reflects the complexity of the food supply and in particular the influence of processed, manufactured foods that may have added vitamins and minerals as well as sugar in their ingredients lists. Alexy et $a l{ }^{(33)}$ has suggested that nutrient fortification of foods may mask the real magnitude of the association between intakes of added sugars and micronutrients. The issue may be of relevance to our study as many breakfast cereal products in Australia include added vitamins and minerals in their ingredient lists and were classified as 'core' foods ${ }^{(34-36)}$, although at the same time contain significant amount of added sugars ${ }^{(37)}$. To put this in perspective, a sub-analysis of the 2007ANCNPAS found that breakfast cereals (including breakfast cereal bars) provided approximately $6 \cdot 4 \%$ of all added sugars consumed by the respondents of 2007ANCNPAS ${ }^{(38)}$; so the impact would only be incremental. Fortification, however, may have less of an impact on nutrients in general as Australia has very strict food standards, which limits the types of foods that could be fortified ${ }^{(39)}$.

The inconsistent direction of associations between \%ETS and the likelihood of not meeting the NRV highlights the limitation of using total sugars as a marker of diet quality. Our results showed a reduced likelihood of not meeting the NRV for $\mathrm{Ca}$, vitamin $\mathrm{C}, \mathrm{K}, \mathrm{I}$ and $\mathrm{Na}$ when \%ETS increased. These associations, apart from that for $\mathrm{Na}$, are likely a result of the coexistence of natural sugars and these nutrient in foods, such as $\mathrm{Ca}$ and $\mathrm{I}$ in dairy foods (a source of lactose), vitamin $\mathrm{C}$ and $\mathrm{K}$ in fruits with natural sugars, etc. However, the observation that subjects with higher \%ETS are less likely to exceed to UL of $\mathrm{Na}$ is interesting. A possible explanation lies in the characteristics of high-salt foods, that most of them are low in sugar - for example, potato crisps and processed meat.

Moving to the food pattern analysis is informative. Clearly, an increase in added sugar intake was consistently associated with a decrease in intake of nutrient-dense 'core' foods such as vegetables, dairy foods, meat and fruit, although being positively associated with the intake of nutrient-poor 'extra' foods such as cakes, biscuits, sugar-sweetened beverages, savoury snacks and confectionery. With this in mind, it suggests that limiting the intake of energy-dense nutrient-poor foods with high levels of added sugar may improve diet quality, which is supported by our findings when the sources of added sugars (from 'core' food $v$. from 'extra' foods) were taken into account. In contrast, the natural sugar content of some nutrient-rich foods is implicated in the results for the total sugars analyses. The positive association between \%ETS and intakes of fruit $\left(P_{\text {trend }}<0.001\right)$ and dairy products $\left(P_{\text {trend }}<0.001\right)$ confirms the inherent limitation of using total sugars in analyses of diet quality, as explained above.

From a beverages perspective, there is likely to be an even greater issue with variation in nutrient content. The subgroup analysis revealed that \%EAS was strongly positively associated with the intake of sugar-sweetened beverages, whereas negatively associated with intakes of other beverages. This suggests that sugar-sweetened beverages are significant contributors to the intakes of added sugars (as \%energy), and they may displace other beverages with low added sugar contents such as 
Table 5. Mean consumption level of various food groups according to age- and sex-specific quintiles (Q) of usual* percentage energy (\%E) from added sugars (Mean values and $95 \%$ confidence intervals; $\beta$ coefficients with their standard errors)

\begin{tabular}{|c|c|c|c|c|c|c|c|c|c|c|c|c|c|c|}
\hline \multirow[b]{3}{*}{ Food groups } & \multicolumn{10}{|c|}{$\% \mathrm{E}$ from added sugars } & \multirow[b]{3}{*}{$\beta \dagger$} & \multirow[b]{3}{*}{ SE } & \multirow[b]{3}{*}{$R^{2}$} & \multirow[b]{3}{*}{$P_{\text {trend }} \dagger$} \\
\hline & \multicolumn{2}{|r|}{ Q1 } & \multicolumn{2}{|r|}{ Q2 } & \multicolumn{2}{|r|}{ Q3 } & \multicolumn{2}{|r|}{ Q4 } & \multicolumn{2}{|r|}{ Q5 } & & & & \\
\hline & Mean & $95 \% \mathrm{Cl}$ & Mean & $95 \% \mathrm{Cl}$ & Mean & $95 \% \mathrm{Cl}$ & Mean & $95 \% \mathrm{Cl}$ & Mean & $95 \% \mathrm{Cl}$ & & & & \\
\hline \multicolumn{15}{|l|}{ 'Core' food } \\
\hline Non-alcoholic be & $1156 \cdot 8$ & $1116 \cdot 4,1197 \cdot 2$ & 1152.5 & $1111.9,1193 \cdot 1$ & $1110 \cdot 0$ & $1069.5,1150 \cdot 4$ & $1047 \cdot 8$ & $1007 \cdot 6,1087 \cdot 9$ & 985.5 & $945 \cdot 3,1025 \cdot 7$ & -18.4 & 2.5 & 0.143 & $<0.001$ \\
\hline Fruit and vegetable juices (g) & 176.6 & $162 \cdot 0,191 \cdot 2$ & 171.9 & $157 \cdot 2,186.6$ & $163 \cdot 0$ & $148 \cdot 3,177 \cdot 6$ & 141.9 & $127 \cdot 5,156 \cdot 4$ & $136 \cdot 2$ & $121 \cdot 7,150 \cdot 7$ & -4.4 & 0.9 & 0.027 & $<0.001$ \\
\hline Cereal grains and products $(\mathrm{g}) \S$ & 375.8 & $363.4,388.3$ & $336 \cdot 7$ & $324 \cdot 2,349 \cdot 2$ & 317.9 & $305.4,330.4$ & 292.0 & $279.6,304 \cdot 3$ & $244 \cdot 3$ & $231 \cdot 9,256 \cdot 7$ & $-12 \cdot 9$ & 0.8 & 0.202 & $<0.001$ \\
\hline Cereal-based products $(\mathrm{g}) \|$ & $57 \cdot 8$ & $49 \cdot 2,66 \cdot 4$ & $72 \cdot 1$ & $63 \cdot 5,80 \cdot 8$ & 62.5 & $53.9,71.7$ & $73 \cdot 1$ & $64.5,81.6$ & 67.5 & $58.9,76.0$ & 0.5 & 0.5 & 0.032 & 0.341 \\
\hline Fish, sea foods and products ( $\mathrm{g}$ ) & 30.4 & $26 \cdot 2,34 \cdot 5$ & 25.5 & $21 \cdot 3,29 \cdot 7$ & 29.1 & $24 \cdot 9,33.3$ & $19 \cdot 0$ & $14 \cdot 9,23 \cdot 2$ & $17 \cdot 6$ & $13 \cdot 5,21 \cdot 8$ & -1.4 & 0.3 & 0.013 & $<0.001$ \\
\hline Fruits and fruit-based products (g) & 296.7 & $282.8,310.6$ & $280 \cdot 7$ & $266 \cdot 7,294 \cdot 7$ & $267 \cdot 1$ & $253 \cdot 2,281 \cdot 1$ & 233.6 & $219 \cdot 7,247 \cdot 4$ & $192 \cdot 3$ & $178 \cdot 4,206 \cdot 1$ & $-10 \cdot 9$ & 0.8 & 0.070 & $<0.001$ \\
\hline Eggs and egg-based products (g) & 16.5 & $14.1,18.9$ & $14 \cdot 8$ & $12 \cdot 4,17 \cdot 2$ & 14.1 & $11 \cdot 7,16 \cdot 4$ & $13 \cdot 6$ & $11 \cdot 2,15 \cdot 9$ & 11.9 & $9 \cdot 6,14 \cdot 2$ & -0.5 & 0.1 & 0.007 & $<0.001$ \\
\hline Meat, poultry and game and their products (g) & 206.8 & $196.7,216.9$ & 189.9 & $179 \cdot 8,200 \cdot 0$ & $195 \cdot 1$ & $185 \cdot 0,205 \cdot 2$ & 187.4 & $177.4,197.4$ & $170 \cdot 4$ & $160 \cdot 4,180 \cdot 4$ & $-3 \cdot 4$ & 0.6 & 0.207 & $<0.001$ \\
\hline Dairy products $(\mathrm{g}) \boldsymbol{\Pi}$ & 464.7 & $442 \cdot 8,486 \cdot 6$ & $496 \cdot 8$ & $474 \cdot 8,518 \cdot 8$ & 463.8 & $441 \cdot 8,485 \cdot 7$ & 454.2 & $432 \cdot 4,476 \cdot 0$ & 394.0 & $372 \cdot 2,415 \cdot 8$ & $-8 \cdot 2$ & 1.3 & 0.124 & $<0.001$ \\
\hline Dairy products (serves)ף & 1.3 & $1 \cdot 2,1 \cdot 3$ & 1.4 & $1.3,1.4$ & 1.4 & $1.3,1.5$ & 1.3 & $1 \cdot 3,1.4$ & 1.3 & $1 \cdot 2,1 \cdot 3$ & -0.0 & 0.0 & 0.120 & 0.305 \\
\hline Dairy alternatives (g) & 13.2 & $6 \cdot 9,19 \cdot 4$ & 14.9 & $8 \cdot 6,21 \cdot 2$ & $16 \cdot 3$ & $10 \cdot 1,22 \cdot 6$ & $17 \cdot 1$ & $10 \cdot 9,23 \cdot 3$ & $10 \cdot 9$ & $4 \cdot 7,17 \cdot 1$ & -0.2 & 0.4 & 0.003 & 0.507 \\
\hline Soups (g) & 61.6 & $51.8,71.4$ & $56 \cdot 7$ & $46 \cdot 8,66 \cdot 5$ & 39.2 & $29.4,49.0$ & 35.7 & $26 \cdot 0,45 \cdot 5$ & 29.6 & $19.9,39.4$ & -3.3 & 0.6 & 0.021 & $<0.001$ \\
\hline Seeds and nut & 5.9 & $5 \cdot 0,6 \cdot 8$ & $5 \cdot 1$ & $4 \cdot 2,6 \cdot 0$ & 4.8 & $3 \cdot 9,5 \cdot 7$ & 4.2 & $3 \cdot 3,5 \cdot 1$ & 2.8 & $1.9,3.7$ & -0.3 & 0.1 & 0.023 & $<0.001$ \\
\hline Sauces $(\mathrm{g})^{* *}$ & $25 \cdot 7$ & $22 \cdot 2,29 \cdot 1$ & 19.9 & $16 \cdot 4,23 \cdot 3$ & 22.4 & $19 \cdot 0,25 \cdot 9$ & 20.7 & $17 \cdot 3,24 \cdot 2$ & 19.2 & $15 \cdot 7,22 \cdot 6$ & -0.6 & 0.2 & 0.022 & 0.008 \\
\hline Vegetables $(\mathrm{g}) \dagger \dagger$ & $242 \cdot 3$ & $230.7,253.9$ & $212 \cdot 6$ & $200 \cdot 0,224 \cdot 2$ & $203 \cdot 2$ & $191 \cdot 6,214.8$ & 190.8 & $179 \cdot 3,202 \cdot 4$ & 176.5 & $164 \cdot 9,188.0$ & $-6 \cdot 3$ & 0.7 & 0.072 & $<0.001$ \\
\hline Legumes and legume- $b$ & $19 \cdot 6$ & $15 \cdot 9,23 \cdot 3$ & 18.5 & $14 \cdot 8,22 \cdot 2$ & 14.9 & $11 \cdot 2,18 \cdot 6$ & 11.5 & $7 \cdot 8,15 \cdot 2$ & 7.4 & $3 \cdot 8,11 \cdot 1$ & $-1 \cdot 2$ & 0.2 & 0.009 & $<0.001$ \\
\hline \multicolumn{15}{|l|}{ 'Extra' foods } \\
\hline Non-alcoholic b & 153.4 & $130 \cdot 2,176 \cdot 7$ & $211 \cdot 2$ & $197 \cdot 8,244.5$ & 282.5 & $259 \cdot 2,305 \cdot 7$ & 389.4 & $366 \cdot 3,412 \cdot 5$ & $577 \cdot 3$ & $554 \cdot 2,600 \cdot 4$ & 43.9 & 1.4 & 0.363 & $<0.001$ \\
\hline Tea and coffee $(\mathrm{g})$ & $28 \cdot 7$ & $20.5,36 \cdot 9$ & $37 \cdot 2$ & $29 \cdot 0,45 \cdot 5$ & 33.9 & $25 \cdot 7,42 \cdot 1$ & $35 \cdot 3$ & $27 \cdot 1,43 \cdot 4$ & $40 \cdot 0$ & $31 \cdot 9,48 \cdot 2$ & -0.1 & 0.5 & 0.059 & 0.825 \\
\hline Sugar-sweetened bev & 90.6 & $69 \cdot 8,111 \cdot 3$ & 157.3 & $136 \cdot 4,178 \cdot 1$ & 215.9 & $195 \cdot 2,236 \cdot 7$ & $329 \cdot 3$ & $308.6,349.9$ & 506.9 & $486 \cdot 3,527 \cdot 6$ & 44.5 & 1.2 & 0.356 & $<0.001$ \\
\hline rages $(\mathrm{g})$ & 34.2 & $26 \cdot 3,42 \cdot 0$ & $26 \cdot 7$ & $18.8,34.5$ & $32 \cdot 6$ & $24.8,40.4$ & 24.9 & $17 \cdot 1,32 \cdot 6$ & 30.4 & $22 \cdot 6,38 \cdot 2$ & -0.5 & 0.5 & 0.034 & 0.306 \\
\hline Cereal grains and products $(\mathrm{g}) \S$ & 3.1 & $1.8,4.5$ & 5.5 & $4.2,6 \cdot 8$ & 2.9 & $1 \cdot 6,4 \cdot 2$ & 4.0 & $2 \cdot 7,5 \cdot 3$ & 4.7 & $3.4,6 \cdot 0$ & 0.1 & 0.1 & 0.005 & 0.394 \\
\hline Cereal-based products $(\mathrm{g}) \|$ & $127 \cdot 0$ & $117 \cdot 6,136 \cdot 5$ & $129 \cdot 7$ & $120 \cdot 2,139 \cdot 3$ & 141.9 & $132.4,151.4$ & $145 \cdot 9$ & $136 \cdot 5,155 \cdot 3$ & 156.0 & $146 \cdot 6,165 \cdot 4$ & 3.6 & 0.6 & 0.202 & $<0.001$ \\
\hline Fats and oils (g) & $12 \cdot 8$ & $12 \cdot 0,13 \cdot 5$ & $12 \cdot 2$ & $11.4,12.9$ & $11 \cdot 2$ & $10 \cdot 4,12 \cdot 0$ & 11.5 & $10 \cdot 7,12 \cdot 3$ & $10 \cdot 3$ & $9.5,11 \cdot 1$ & -0.3 & 0.0 & 0.031 & $<0.001$ \\
\hline id game and their products ( $\mathrm{g}$ ) & 1.6 & $1 \cdot 1,2 \cdot 1$ & 1.5 & $1.0,2 \cdot 0$ & 1.0 & $0.5,1.5$ & 1.6 & $1 \cdot 1,2 \cdot 1$ & 0.9 & $0.4,1.3$ & -0.1 & 0.0 & 0.006 & 0.014 \\
\hline Dairy products $(\mathrm{g}) \boldsymbol{\Phi}$ & $26 \cdot 7$ & $19 \cdot 4,34.0$ & 53.4 & $46 \cdot 1,60 \cdot 8$ & $71 \cdot 2$ & $63.9,78.5$ & $70 \cdot 2$ & $63.0,77.5$ & $97 \cdot 7$ & $90 \cdot 4,105 \cdot 0$ & $6 \cdot 1$ & 0.5 & 0.075 & $<0.001$ \\
\hline Sauce & $15 \cdot 6$ & $13 \cdot 3,18 \cdot 0$ & 14.4 & $12 \cdot 0,16 \cdot 8$ & $19 \cdot 3$ & $16 \cdot 9,21 \cdot 6$ & $17 \cdot 8$ & $15 \cdot 5,20 \cdot 2$ & $16 \cdot 7$ & $14 \cdot 3,19 \cdot 0$ & 0.2 & 0.1 & 0.044 & 0.118 \\
\hline Vegetables $(\mathrm{g}) \dagger \dagger$ & 33.0 & $28.5,37.5$ & $34 \cdot 1$ & $29 \cdot 6,38.6$ & 34.5 & $30 \cdot 1,39 \cdot 0$ & 39.6 & $35 \cdot 1,44 \cdot 0$ & 43.0 & $38 \cdot 6,47.5$ & $1 \cdot 1$ & 0.3 & 0.053 & $<0.001$ \\
\hline Savoury snacks (g) & 18.4 & $16 \cdot 1,20 \cdot 6$ & 17.5 & $15 \cdot 3,19 \cdot 8$ & 18.4 & $16 \cdot 1,20 \cdot 6$ & $18 \cdot 3$ & $16 \cdot 1,20 \cdot 5$ & 18.4 & $16 \cdot 2,20 \cdot 6$ & 0.2 & 0.1 & 0.045 & 0.248 \\
\hline Sugars (g) & $13 \cdot 3$ & $9 \cdot 5,17 \cdot 1$ & $23 \cdot 8$ & $20 \cdot 0,27 \cdot 6$ & 28.0 & $24 \cdot 2,31 \cdot 8$ & 33.1 & $29.4,36.9$ & $51 \cdot 3$ & $47 \cdot 6,55 \cdot 1$ & 3.5 & 0.2 & 0.060 & $<0.001$ \\
\hline Confectionery (g) & $15 \cdot 1$ & $12 \cdot 2,17 \cdot 9$ & $21 \cdot 2$ & $18 \cdot 3,24.1$ & $27 \cdot 2$ & $24 \cdot 3,30 \cdot 0$ & 38.2 & $35 \cdot 4,41 \cdot 1$ & 56.6 & $53.7,59.4$ & 3.9 & 0.2 & 0.164 & $<0.001$ \\
\hline
\end{tabular}

Values are estimated marginal means calculated using ANCOVA, with total energy intake and BMI z-scores as covariates except for energy, where only BMI $z$-score was included as a covariate.

* Usual intake calculated using the multiple source method ${ }^{(27)}$

$\dagger \beta, R^{2}$ and $P_{\text {trend }}$ calculated using linear regression with the food group intake as the dependent variable, \%E from added sugars as a continuous independent variable and age, sex, total energy intake and BMI $z$-scores as covariates. ‡ 'Core' non-alcoholic beverages include: fruit and vegetable juices, plain or mineral water and beverage flavourings made up with milk; 'extra' non-alcoholic beverages include: tea and coffee, fruit drinks, cordial/mixers, carbonated soft drinks, flavoured water, electrolyte drinks, energy drinks and beverage flavourings (dry or made up with water).

$\S$ 'Core' cereal grains and products include: plain grains, plain breads, low fat-filled/topped bread, low-sugar sweet buns/scrolls, flat breads, low-fat tortilla and all breakfast cereals; 'extra' cereal grains and products include: higher fat-filled/ topped breads, higher sugar sweet buns/scrolls, higher-fat tortilla/taco and high-fat noodles.

'Core' cereal-based products include: low-fat savoury biscuits, rice and maize crackers/cakes, low sugar scones, low-fat sandwiches, pasta or noodle dishes, low-sugar/fat waffles and batter-based products and crumpet; 'extra' cerealbased products include: sweet biscuits, high-fat savoury biscuits, cakes and slices, higher sugar scones, cereal-based desserts, pastries, pizza, higher-fat sandwiches, hamburgers, taco/tortilla based dishes, savoury dumplings, highersugar/fat batter-based products and doughnuts.

I 'Core' dairy products include: fluid milk including lower fat-/sugar-flavoured milk, yoghurts, cheese, lower-fat ice-creams and lower-fat custard; 'extra' dairy products include: condensed milk, cream, higher-fat ice-creams, frozen yoghurts, higher-fat custards, dairy desserts and higher-fat/sugar flavoured milk; dairy servings defined as follows: $250 \mathrm{~g}$ milk (including flavoured milk); $200 \mathrm{~g}$ yoghurt or custards; $40 \mathrm{~g}$ cheese; and $100 \mathrm{~g}$ ice cream,

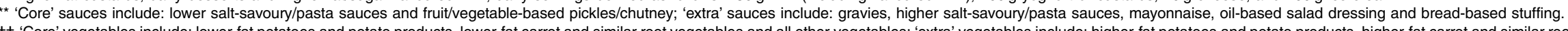
vegetables. 
Table 6. Mean consumption level of various food groups according to age- and sex-specific quintiles $(\mathrm{Q})$ of usual ${ }^{\star}$ percentage energy (\%E) from total sugars (Mean values and $95 \%$ confidence intervals; $\beta$ coefficients with their standard errors)

\begin{tabular}{|c|c|c|c|c|c|c|c|c|c|c|c|c|c|c|}
\hline \multirow[b]{3}{*}{ Food groups } & \multicolumn{10}{|c|}{$\% \mathrm{E}$ from total sugars } & \multirow[b]{3}{*}{$\beta \dagger$} & \multirow[b]{3}{*}{ SE } & \multirow[b]{3}{*}{$R^{2}$} & \multirow[b]{3}{*}{$P_{\text {trend }} \dagger$} \\
\hline & \multicolumn{2}{|r|}{ Q1 } & \multicolumn{2}{|r|}{ Q2 } & \multicolumn{2}{|r|}{ Q3 } & \multicolumn{2}{|r|}{ Q4 } & \multicolumn{2}{|r|}{ Q5 } & & & & \\
\hline & Mean & $95 \% \mathrm{Cl}$ & Mean & $95 \% \mathrm{Cl}$ & Mean & $95 \% \mathrm{Cl}$ & Mean & $95 \% \mathrm{Cl}$ & Mean & $95 \% \mathrm{Cl}$ & & & & \\
\hline \multicolumn{15}{|l|}{ 'Core' food } \\
\hline Non-alcoholic beverages $(\mathrm{g}) \ddagger$ & $1119 \cdot 3$ & $1078 \cdot 4,1160 \cdot 2$ & $1069 \cdot 8$ & $1029 \cdot 3,1110 \cdot 2$ & 1073.9 & $1033.5,1114.2$ & $1094 \cdot 2$ & $1053 \cdot 7,1134 \cdot 7$ & 1094.0 & $1053 \cdot 5,1134.7$ & $1 \cdot 1$ & $2 \cdot 3$ & 0.132 & 0.613 \\
\hline Fruit and vegetable juices (g) & $90 \cdot 2$ & $75 \cdot 9,104.6$ & $131 \cdot 1$ & $116 \cdot 9,145 \cdot 4$ & 149.8 & $135.6,164.0$ & 189.1 & $174 \cdot 8,203 \cdot 3$ & 228.7 & $214.4,243.0$ & $12 \cdot 8$ & 0.8 & 0.080 & $<0.001$ \\
\hline Cereal grains and products $(\mathrm{g}) \S$ & 375.5 & $362 \cdot 9,388 \cdot 1$ & 331.3 & $318.8,343.8$ & $306 \cdot 2$ & $293 \cdot 8,318 \cdot 7$ & $292 \cdot 2$ & $279 \cdot 7,304.7$ & $260 \cdot 8$ & $248 \cdot 2,273 \cdot 4$ & -9.8 & 0.7 & 0.186 & $<0.001$ \\
\hline Cereal-based products $(\mathrm{g}) \|$ & 73.6 & $64 \cdot 9,82 \cdot 2$ & 63.6 & $55 \cdot 1,72 \cdot 2$ & $61 \cdot 1$ & $52.5,69 \cdot 6$ & 67.9 & $59 \cdot 4,76.5$ & $66 \cdot 9$ & $58 \cdot 3,75 \cdot 6$ & -0.4 & 0.5 & 0.032 & 0.379 \\
\hline Fish, sea foods and products (g) & 35.5 & $31.3,39.6$ & $25 \cdot 1$ & $21 \cdot 0,29 \cdot 3$ & 21.9 & $17 \cdot 8,26 \cdot 1$ & 19.4 & $15 \cdot 2,23 \cdot 6$ & $19 \cdot 7$ & $15 \cdot 6,23 \cdot 9$ & $-1 \cdot 7$ & 0.2 & 0.018 & $<0.001$ \\
\hline Fruits and fruit-based products $(\mathrm{g})$ & $178 \cdot 7$ & $164 \cdot 8,192.5$ & $222 \cdot 4$ & $208 \cdot 7,236 \cdot 1$ & 255.7 & $242 \cdot 0,269 \cdot 4$ & 299.6 & $285 \cdot 9,313 \cdot 3$ & $312 \cdot 7$ & $298 \cdot 9,326 \cdot 5$ & 12.5 & 0.8 & 0.094 & $<0.001$ \\
\hline Eggs and egg-based products (g) & 17.9 & $15 \cdot 5,20 \cdot 2$ & $16 \cdot 2$ & $13.8,18.5$ & $13 \cdot 6$ & $11 \cdot 3,16 \cdot 0$ & $12 \cdot 3$ & $10 \cdot 0,14.7$ & $10 \cdot 8$ & $8 \cdot 5,13.2$ & -0.7 & 0.1 & 0.010 & $<0.001$ \\
\hline Meat, poultry and game and their products $(\mathrm{g})$ & 228.4 & $218.4,238.4$ & 209.5 & $199 \cdot 6,219 \cdot 4$ & $185 \cdot 8$ & $176 \cdot 0,195 \cdot 7$ & $176 \cdot 3$ & $166 \cdot 4,186 \cdot 2$ & 149.5 & $139.5,159.4$ & -6.5 & 0.6 & 0.226 & $<0.001$ \\
\hline Dairy products $(\mathrm{g}) \boldsymbol{\uparrow}$ & $315 \cdot 1$ & $293 \cdot 6,336 \cdot 7$ & 435.5 & $414 \cdot 2,456 \cdot 8$ & 483.3 & $462 \cdot 0,504 \cdot 6$ & $509 \cdot 6$ & $488 \cdot 2,531 \cdot 0$ & $527 \cdot 6$ & $506 \cdot 1,549 \cdot 0$ & $17 \cdot 1$ & 1.2 & 0.161 & $<0.001$ \\
\hline Dairy products (serves) & 1.1 & $1 \cdot 0,1 \cdot 1$ & 1.3 & $1.2,1.3$ & 1.4 & $1.3,1.4$ & 1.4 & $1.4,1.5$ & 1.5 & $1.4,1.5$ & 0.0 & 0.0 & 0.144 & $<0.001$ \\
\hline Dairy alternatives (g) & $27 \cdot 3$ & $21 \cdot 0,33 \cdot 6$ & $17 \cdot 1$ & $10 \cdot 9,23 \cdot 3$ & $11 \cdot 2$ & $5 \cdot 0,17 \cdot 4$ & $12 \cdot 2$ & $6 \cdot 0,18.4$ & 4.6 & $-1 \cdot 7,10 \cdot 8$ & -1.4 & 0.3 & 0.008 & $<0.001$ \\
\hline Soups $(\mathrm{g})$ & 55.6 & $45 \cdot 7,65 \cdot 5$ & 41.3 & $31.5,51 \cdot 1$ & 41.2 & $31.4,50 \cdot 9$ & $48 \cdot 2$ & $38 \cdot 4,58 \cdot 0$ & $36 \cdot 3$ & $26 \cdot 5,46 \cdot 2$ & -1.0 & 0.5 & 0.015 & 0.082 \\
\hline Seeds and nuts and their products ( $\mathrm{g}$ ) & 5.0 & $4.1,5.9$ & $6 \cdot 1$ & $5 \cdot 2,7.0$ & 4.4 & $3.5,5 \cdot 3$ & 4.0 & $3.1,4.9$ & 3.3 & $2 \cdot 4,4 \cdot 2$ & -0.2 & 0.0 & 0.019 & $<0.001$ \\
\hline Sauces $(\mathrm{g})^{\star \star}$ & $25 \cdot 2$ & $21 \cdot 7,28 \cdot 7$ & $19 \cdot 1$ & $15 \cdot 6,22 \cdot 5$ & 21.6 & $18 \cdot 2,25 \cdot 1$ & $23 \cdot 7$ & $20 \cdot 3,27 \cdot 2$ & $18 \cdot 2$ & $14 \cdot 8,21 \cdot 7$ & -0.2 & 0.2 & 0.055 & 0.195 \\
\hline Vegetables $(\mathrm{g}) \dagger \dagger$ & 207.9 & $196 \cdot 1,219 \cdot 7$ & $207 \cdot 0$ & $195 \cdot 4,218 \cdot 7$ & 204.6 & $193 \cdot 0,216 \cdot 2$ & $207 \cdot 1$ & $195.5,218.8$ & $198 \cdot 3$ & $186 \cdot 6,210 \cdot 1$ & -0.4 & 0.6 & 0.055 & 0.505 \\
\hline Legumes and legume-based products (g) & $17 \cdot 0$ & $13 \cdot 3,20 \cdot 7$ & $18 \cdot 2$ & $14.5,21.9$ & 14.7 & $11 \cdot 1,18 \cdot 4$ & $14 \cdot 0$ & $10 \cdot 3,17 \cdot 7$ & 7.8 & $4 \cdot 1,11.5$ & -0.8 & 0.2 & 0.006 & $<0.001$ \\
\hline \multicolumn{15}{|l|}{ ‘Extra' foods } \\
\hline Non-alcoholic beverages $(\mathrm{g}) \ddagger$ & 234.1 & $209 \cdot 2,259 \cdot 0$ & 268.4 & $243 \cdot 8,293 \cdot 0$ & $307 \cdot 7$ & $283 \cdot 1,332 \cdot 3$ & $346 \cdot 0$ & $321 \cdot 3,370 \cdot 6$ & 471.9 & $447 \cdot 1,496 \cdot 7$ & $20 \cdot 2$ & 1.4 & 0.251 & $<0.001$ \\
\hline Tea and coffee $(\mathrm{g})$ & 38.6 & $30 \cdot 4,46 \cdot 8$ & 34.8 & $26 \cdot 7,43 \cdot 0$ & $40 \cdot 1$ & $32 \cdot 0,48 \cdot 2$ & 33.2 & $25 \cdot 0,41 \cdot 4$ & $28 \cdot 3$ & $20 \cdot 1,36 \cdot 5$ & -0.6 & 0.5 & 0.060 & 0.186 \\
\hline Sugar-sweetened beverages ( $\mathrm{g}$ ) & $155 \cdot 2$ & $132 \cdot 8,177 \cdot 6$ & $205 \cdot 7$ & $183 \cdot 5,227 \cdot 8$ & 244.4 & $222 \cdot 3,266 \cdot 5$ & $285 \cdot 3$ & $263 \cdot 1,307 \cdot 4$ & $413 \cdot 6$ & $391.3,435.9$ & 21.9 & $1 \cdot 2$ & 0.216 & $<0.001$ \\
\hline Intensely sweetened beverages $(\mathrm{g})$ & $40 \cdot 3$ & $32 \cdot 4,48 \cdot 2$ & 27.9 & $20 \cdot 1,35 \cdot 7$ & $23 \cdot 2$ & $15.4,31.0$ & 27.5 & $19 \cdot 7,35 \cdot 3$ & 30.0 & $22 \cdot 1,37 \cdot 8$ & $-1 \cdot 1$ & 0.4 & 0.035 & 0.012 \\
\hline Cereal grains and products $(\mathrm{g}) \S$ & 4.9 & $3 \cdot 6,6 \cdot 2$ & $5 \cdot 2$ & $3.9,6.5$ & 3.6 & $2 \cdot 2,4.9$ & $3 \cdot 1$ & $1 \cdot 8,4 \cdot 4$ & 3.5 & $2 \cdot 2,4 \cdot 8$ & -0.2 & 0.1 & 0.007 & 0.004 \\
\hline Cereal-based products $(\mathrm{g}) \|$ & 148.8 & $139.3,158.4$ & 141.6 & $132 \cdot 1,151 \cdot 0$ & 144.5 & $135 \cdot 1,153 \cdot 9$ & $122 \cdot 9$ & $113 \cdot 4,132 \cdot 3$ & $143 \cdot 2$ & $133.7,152 \cdot 7$ & -1.6 & 0.5 & 0.197 & 0.002 \\
\hline Fats and oils $(\mathrm{g})$ & 13.4 & $12 \cdot 6,14 \cdot 2$ & 12.5 & $11 \cdot 7,13 \cdot 3$ & 12.6 & $11.8,13.3$ & $10 \cdot 6$ & $9.8,11 \cdot 3$ & 8.9 & $8 \cdot 1,9 \cdot 7$ & -0.4 & 0.0 & 0.046 & $<0.001$ \\
\hline Meat, poultry and game and their products (g) & 1.8 & $1 \cdot 3,2 \cdot 3$ & $2 \cdot 1$ & $1 \cdot 6,2.5$ & 0.9 & $0.5,1.4$ & $1 \cdot 1$ & $0.6,1.6$ & 0.6 & $0.1,1 \cdot 2$ & -0.1 & 0.0 & 0.009 & $<0.001$ \\
\hline Dairy products $(\mathrm{g}) \boldsymbol{\Pi}$ & 41.5 & $34 \cdot 0,49 \cdot 0$ & $59 \cdot 0$ & $51 \cdot 6,66 \cdot 3$ & $62 \cdot 2$ & $54.8,69.6$ & 68.0 & $60.6,75.5$ & 88.9 & $81.5,96 \cdot 4$ & 4.0 & 0.4 & 0.056 & $<0.001$ \\
\hline Sauces $(\mathrm{g})^{\star *}$ & $16 \cdot 4$ & $14 \cdot 0,18 \cdot 8$ & 17.4 & $15 \cdot 0,19 \cdot 7$ & 17.6 & $15 \cdot 3,20 \cdot 0$ & $15 \cdot 7$ & $13 \cdot 4,18 \cdot 1$ & $16 \cdot 6$ & $14 \cdot 2,19 \cdot 0$ & -0.1 & 0.1 & 0.044 & 0.549 \\
\hline Vegetables $(\mathrm{g}) \dagger$ & 43.4 & $38.9,47.9$ & 40.9 & $36 \cdot 4,45 \cdot 3$ & $42 \cdot 7$ & $38.3,47 \cdot 1$ & 29.5 & $25.0,33.9$ & $27 \cdot 8$ & $23 \cdot 3,32 \cdot 3$ & -1.5 & 0.3 & 0.057 & $<0.001$ \\
\hline Savoury snacks (g) & $26 \cdot 0$ & $23 \cdot 8,28 \cdot 3$ & $17 \cdot 1$ & $14 \cdot 9,19 \cdot 4$ & 16.9 & $14.7,19 \cdot 1$ & $17 \cdot 2$ & $15 \cdot 0,19 \cdot 4$ & 13.8 & $11.5,16 \cdot 0$ & -0.9 & 0.1 & 0.056 & $<0.001$ \\
\hline Sugars (g) & $16 \cdot 5$ & $12 \cdot 7,20 \cdot 3$ & 21.4 & $17 \cdot 6,25 \cdot 1$ & $31 \cdot 2$ & $27.4,34.9$ & 33.0 & $29 \cdot 2,36 \cdot 8$ & 47.9 & $44 \cdot 0,51 \cdot 7$ & $2 \cdot 8$ & 0.2 & 0.049 & $<0.001$ \\
\hline Confectionery (g) & $19 \cdot 1$ & $16 \cdot 1,22 \cdot 1$ & $25 \cdot 8$ & $22 \cdot 8,28 \cdot 7$ & 33.2 & $30 \cdot 3,36 \cdot 2$ & 37.5 & $34.5,40.5$ & 42.9 & $39.9,45.9$ & 2.0 & 0.2 & 0.097 & $<0.001$ \\
\hline
\end{tabular}

Values are estimated marginal means calculated using ANCOVA, with total energy intake and BMI z-scores as covariates except for energy, where only BMI $z$-score was included as a covariate.

* Usual intake calculated using the multiple source method ${ }^{(27}$.

$\dagger \beta$ (sE), $R^{2}$ and $P_{\text {trend }}$ calculated using linear regression with the food group intake as the dependent variable, \%E from added sugars as a continuous independent variable and age, sex, total energy intake and BMI $z$-scores as covariates. ‡ 'Core' non-alcoholic beverages include: fruit and vegetable juices, plain or mineral water and beverage flavourings made up with milk; 'extra' non-alcoholic beverages include: tea and coffee, fruit drinks, cordial/mixers, carbonated soft drinks, flavoured water, electrolyte drinks, energy drinks and beverage flavourings (dry or made up with water).

$\S$ 'Core' cereal grains and products include: plain grains, plain breads, low fat-filled/topped bread, low-sugar sweet buns/scrolls, flat breads, low-fat tortilla and all breakfast cereals; 'extra' cereal grains and products include: higher fat-filled/ topped breads, higher-sugar sweet buns/scrolls, higher-fat tortilla/taco and high-fat noodles,

'Core' cereal-based products include: low fat-savoury biscuits, rice and maize crackers/cakes, low sugar scones, low-fat sandwiches, pasta or noodle dishes, low-sugar/fat waffles and batter-based products and crumpet; 'extra' cerealbased products include: sweet biscuits, high-fat savoury biscuits, cakes and slices, higher sugar scones, cereal-based desserts, pastries, pizza, higher-fat sandwiches, hamburgers, taco-/tortilla-based dishes, savoury dumplings, higher sugar/fat batter-based products and doughnuts.

If 'Core' dairy products include: fluid milk including lower fat/sugar flavoured milk, yoghurts, cheese, lower-fat ice-creams and lower-fat custard; 'extra' dairy products include: condensed milk, cream, higher fat ice-creams, frozen yoghurts, higher-fat custards, dairy desserts and higher-fat/sugar flavoured milk; dairy servings defined as follows: $250 \mathrm{~g}$ milk (including flavoured milk); $200 \mathrm{~g}$ yoghurt or custards; $40 \mathrm{~g}$ cheese; and $100 \mathrm{~g}$ ice cream.

** 'Core' sauces include. lower salt-savoury/pasta sauces and fruit-/vegetable-based pickles/chutney; 'extra' sauces include: gravies, higher salt savoury/pasta sauces, mayonnaise, oil-based salad dressing and bread-based stuffing. †† 'Core' vegetables include: lower-fat potatoes and potato products, lower-fat carrot and similar root vegetables, and all other vegetables; 'extra' vegetables include: higher-fat potatoes and potato products, higher fat carrot and similar root
vegetables. 
fruit juice. This pattern was less apparent when \%ETS was considered, as fruit juices are high in total sugars.

One limitation of our study is that micronutrients from supplements were not included in the analyses. Although supplements' use has become more common among Australians ${ }^{(40,41)}$, which may have covered the individual from the shortfalls in dietary micronutrient intake, many argue that nutrients from supplements were not well absorbed. In addition, the aim of our study was to assess how dietary added and total sugar intakes were associated with dietary macro- and micronutrient intake. Excluding nutrients from supplements could therefore enable us to identify levels of added or total sugar intake, which may increase the likelihood of inadequate dietary nutrient intake.

Although a common choice for dietary survey, assessing dietary intake by $24-\mathrm{h}$ recalls is not without limitations. It is reliant on the respondent to correctly recall the foods and beverages consumed in the past $24 \mathrm{~h}$. Although various prompts and the use of a multiple-pass method may have partly improved the respondents' ability to recall, this is still subject to memory bias. In addition, data obtained from two 24-h recalls may not capture the habitual intake of an individual as dietary intake is subject to high day-to-day variance ${ }^{(42,43)}$, especially for items that are not frequently consumed. To allow better estimation of habitual intake in young children (6 years or below) using multiple 24-h recalls, it has been shown that up to $9 \mathrm{~d}$ of recalls are required to ensure an $80 \%$ correlation between the observed and true mean nutrient intakes of individuals ${ }^{(44)}$. Two 24-h recalls are the usual choice in national nutrition surveys $^{(45-48)}$ to balance the accuracy of the dietary data collected against respondent burden. Although the use of the $\mathrm{MSM}^{(27)}$ on the two 24-h recalls in this study has accounted for some of the intra-person variability, a more accurate estimation of habitual intake could only be achieved either through combining the food frequency data with the recall data in MSM or by increasing the number of recalls. Unfortunately, these were not available.

The analysis is also limited by general limitations to dietary surveys. It had been previously argued that accurate and reliable dietary assessment in children is especially difficult, regardless of whether the children reported their own intake or parental recall was used. In the 2007ANCNPAS, parental recall of food intake was used for children aged 8 years or below, which is likely to result in under-reporting especially when the reporting parent was working and away from home for a significant period of time $e^{(42,49-52)}$. Our results appear to support this proposition where the energy adjusted nutrient intake from parental reports were generally lower than that were selfreported by the child/adolescent. However, when children report their own food intake, they are also likely to inaccurately recall the type of foods they consume due to unfamiliarity of the food $^{(53)}$ as well as information overload (e.g. large number of foods to report), which is likely to result in omission of foods reported $^{(54)}$. Nonetheless, by using the Goldberg cut-off for specific PAL method ${ }^{(26)}$, we have excluded under- and overreporters on the basis of a scientifically accepted methodology to minimise the effect of under- and over-reporting on the results, although it is acknowledged that the cut-offs were conservative and only extreme degrees of misreporting were identified this way. In addition, this method does not allow the distinction of varying degrees of misreporting, meaning that a clear-cut approach was taken ${ }^{(26)}$.

Seasonality is another limitation of dietary surveys, and the data collection period spanned across autumn and winter, which may have affected intakes of seasonal foods. Ice cream and sugary drinks, for example, may be consumed more during the summer months.

Despite these limitations, the use of a published systematic method to estimate the added sugar content of the food items in AUSNUT2007 was a particular strength of our study. The generalisability of the findings was also increased through the use of a nationally representative sample.

\section{Conclusion}

Using a national survey on Australian children and adolescents, analyses involving intakes of added sugar provided more consistent associations with micronutrient intakes and diet quality compared with assessments of total sugar intake. Higher intakes of added sugar were associated with micronutrient dilution in the diet.

\section{Acknowledgements}

The authors would like to thank the Australian Commonwealth Department of Health and Ageing for providing the survey data via the Australian Social Science Data Archive.

Results of this study were included in a tender for the Australian National Health and Medical Research Council (NHMRC; tender no. 2012/0268). The NHMRC provided written approval for this work to be published, and the authors declare that the NHMRC had no influence on the conclusions drawn. The original data of the 2007 ANCNPAS were collected by the Australian Commonwealth Scientific and Industrial Research Organization and the University of South Australia.

J. C. Y. L. and L. C. T. contributed to the conception of the study. J. C. Y. L. performed the statistical analyses and drafted the manuscript. Both authors critically reviewed and interpreted the data, were involved in the subsequent edits of the manuscript and have read and approved the final manuscript. The authors declare that those who carried out the original analysis and collection of the data bear no responsibility for the further analysis or interpretation included in this manuscript.

This was an entirely independent analysis, with no industry association. There are no conflicts of interest.

\section{Supplementary material}

For supplementary material/s referred to in this article, please visit http://dx.doi.org/10.1017/S0007114515003542

\section{Refrences}

1. Barclay AW \& Brand-Miller J (2011) The Australian paradox: a substantial decline in sugars intake over the same timeframe that overweight and obesity have increased. Nutrients 3, 491-504. 
2. Lustig RH, Schmidt LA \& Brindis CD (2012) Public health: the toxic truth about sugar. Nature $\mathbf{4 8 2}, 27-29$.

3. Cottrell RC (2012) Sugar: an excess of anything can harm. Nature 483, 158.

4. Willett WC \& Ludwig DS (2013) Science souring on sugar. BMJ 346, e8077.

5. Watts G (2013) Sugar and the heart: old ideas revisited. BMJ 346, e7800.

6. Sievenpiper JL, de Souza RJ \& Jenkins DJ (2012) Sugar: fruit fructose is still healthy. Nature $\mathbf{4 8 2}, 470$

7. White JS (2012) Sugar-sweetened beverage link to cardiovascular risk factors is unsupported. Am J Clin Nutr 95, 773; author reply 773-774.

8. Coulston AM \& Johnson RK (2002) Sugar and sugars: myths and realities. J Am Diet Assoc 102, 351-353.

9. Baker CW (2002) Sugar association response to 'Sugar and sugars: myths and realities'. J Am Diet Assoc 102, 776.

10. Ruxton CH, Garceau FJ \& Cottrell RC (1999) Guidelines for sugar consumption in Europe: is a quantitative approach justified? Eur J Clin Nutr 53, 503-513.

11. Johnson RK, Appel LJ, Brands M, et al. (2009) Dietary sugars intake and cardiovascular health: a scientific statement from the American Heart Association. Circulation 120, 1011-1020.

12. Faculty of Public Health of the Royal Colleges of Physicians of the United Kingdom (2007) Sugar - a position statement. http://www. fph.org.uk/uploads/ps_sugar.pdf (accessed January 2013).

13. Fitch C \& Keim KS (2012) Position of the Academy of Nutrition and Dietetics: use of nutritive and nonnutritive sweeteners. J Acad Nutr Diet 112, 739-758.

14. Morenga LT, Mallard S \& Mann J (2013) Dietary sugars and body weight: systematic review and meta-analyses of randomised controlled trials and cohort studies. BMJ 346, e7492.

15. Somerset SM (2003) Refined sugar intake in Australian children. Public Health Nutr 6, 809-813.

16. Forshee RA \& Storey ML (2001) The role of added sugars in the diet quality of children and adolescents. J Am Coll Nutr 20, 32-43.

17. Rennie KL \& Livingstone MBE (2007) Associations between dietary added sugar intake and micronutrient intake: a systematic review. Br J Nutr 97, 832-841.

18. Joyce $\mathrm{T} \&$ G Gibney $\mathrm{MJ}$ (2008) The impact of added sugar consumption on overall dietary quality in Irish children and teenagers. J Hum Nutr Diet 21, 438-450.

19. Cobiac L, Record S, Leppard P, et al. (2003) Sugars in the Australian diet: results from the 1995 national nutrition survey. Nutr Diet 60, 152-173.

20. Livingstone MB \& Rennie KL (2009) Added sugars and micronutrient dilution. Obes Rev 10, 34-40.

21. Green Pool Commodity Specialists (2012) Sugar Consumption in Australia: A Statistical Update . Brisbane, QLD: Green Pool Commodity Specialists.

22. Australian Commonwealth Department of Health and Ageing, Australian Commonwealth Scientific and Research Organization \& University of South Australia (2009) The 2007 National Children's Nutrition and Physical Activity Survey. Canberra, ACT: Australian Social Science Data Archive, The Australian National University.

23. University of South Australia, Australian Commonwealth Scientific and Research Organization \& i-View Pty Ltd (2009) User Guide - 2007 Australian National Children's Nutrition and Physical Activity Survey. http://www.health.gov.au/ internet/main/publishing.nsf/Content/AC3F256C715674D5CA 2574D60000237D/\$File/user-guide-v2.pdf (accessed July 2009).

24. Food Standards Australia New Zealand (2008) AUSNUT2007 Food Composition Database. http://www.foodstandards.gov. $\mathrm{au} /$ science/monitoringnutrients/ausnut/Pages/ausnut2007.aspx (accessed November 2013).
25. Louie JCY, Moshtaghian H, Boylan S, et al. (2015) A systematic methodology to estimate added sugar content of foods. Eur J Clin Nutr 69, 154-161.

26. Goldberg GR, Black AE, Jebb SA, et al. (1991) Critical evaluation of energy intake data using fundamental principles of energy physiology: 1 . Derivation of cut-off limits to identify under-recording. Eur J Clin Nutr 45, 569-581.

27. Harttig U, Haubrock J, Knuppel S, et al. (2011) The MSM program: web-based statistics package for estimating usual dietary intake using the multiple source method. Eur J Clin Nutr 65, S87-S91.

28. Australian Commonwealth Department of Health and Ageing, National Health and Medical Research Council (Australia) (2006) Nutrient Reference Values for Australia and New Zealand Including Recommended Dietary Intakes. Canberra, ACT: NHMRC.

29. National Health and Medical Research Council (Australia) (2013) Australian Dietary Guidelines - Providing the Scientific Evidence for Healthier Australian Diets. Canberra, ACT: NHMRC, DoHA.

30. Rangan AM, Kwan J, Flood VM, et al. (2011) Changes in 'extra' food intake among Australian children between 1995 and 2007. Obes Res Clin Prac 5, e55-e63.

31. Rangan AM, Kwan JSL, Louie JCY, et al. (2011) Changes in core food intake among Australian children between 1995 and 2007. Eur J Clin Nutr 65, 1201-1210.

32. Gibson S \& Boyd A (2009) Associations between added sugars and micronutrient intakes and status: further analysis of data from the National Diet and Nutrition Survey of Young People aged 4 to 18 years. Br J Nutr 101, 100-107.

33. Alexy U, Sichert-Hellert W \& Kersting M (2002) Fortification masks nutrient dilution due to added sugars in the diet of children and adolescents. J Nutr 132, 2785-2791.

34. Dugbaza J \& Cunningham J (2012) Estimates of total dietary folic acid intake in the Australian population following mandatory folic acid fortification of bread. J Nutr Metab 2012, 7.

35. Saunders AV, Craig WJ, Baines SK, et al. (2012) Iron and vegetarian diets. MJA Open 1, 11-16.

36. Food Standards Australia New Zealand (2012) Adding vitamins and minerals to food. http://www.foodstandards.gov.au/ consumerinformation/fortification.cfm (accessed March 2013).

37. Louie JC, Dunford EK, Walker KZ, et al. (2012) Nutritional quality of Australian breakfast cereals. Are they improving? Appetite 59, 464-470.

38. Louie JC, Rangan AM, Flood VM, et al. (2012) Added sugar intake of Australian children and adolescents. Australian New Zealand Obesity Society Annual Scientific Meeting, 18-20 October 2012.

39. Food Standards Australia New Zealand (2013) Australia New Zealand Food Standards Code - Standard 1.3.2 - Vitamins and Minerals. Canberra, ACT: FSANZ.

40. Commonwealth Scientific and Industrial Research Organisation (Australia) (2012) The 2007 Australian National Children's Nutrition and Physical Activity Survey, Vol. 3: Dietary Supplements Consumed. Canberra, ACT: DoHA.

41. Australian Bureau of Statistics (2014) Australian Health Survey: Nutrition First Results - Foods and Nutrients, 2011-2012. Canberra, ACT: ABS.

42. Livingstone MBE \& Robson PJ (2000) Measurement of dietary intake in children. Proc Nutr Soc 59, 279-293.

43. Biro G, Hulshof KF, Ovesen L, et al. (2002) Selection of methodology to assess food intake. Eur J Clin Nutr 56, S25-S32.

44. Erkkola M, Kyttala P, Takkinen HM, et al. (2011) Nutrient intake variability and number of days needed to assess intake in preschool children. Br J Nutr 106, 130-140.

45. Deshmukh-Taskar PR, Nicklas TA, O'Neil CE, et al. (2010) The relationship of breakfast skipping and type of breakfast 
consumption with nutrient intake and weight status in children and adolescents: the National Health and Nutrition Examination Survey 1999-2006. J Am Diet Assoc 110, 869-878.

46. Pan WH, Wu HJ, Yeh CJ, et al. (2011) Diet and health trends in Taiwan: comparison of two nutrition and health surveys from 1993-1996 and 2005-2008. Asia Pac J Clin Nutr 20, 238-250.

47. Parnell W, Wilson N, Alexander D, et al. (2008) Exploring the relationship between sugars and obesity. Public Health Nutr 11, 860-866.

48. Australian Bureau of Statistics (2013) Australian Health Survey: First Results, 2011-2012. Canberra, ACT: ABS

49. Klesges RC, Klesges LM, Brown G, et al. (1987) Validation of the 24-hour dietary recall in preschool children. $J$ Am Diet Assoc 87, 1383-1385.
50. Eck LH, Klesges RC \& Hanson CL (1989) Recall of a child's intake from one meal: are parents accurate? J Am Diet Assoc 89, 784-789.

51. Basch CE, Shea S, Arliss R, et al. (1990) Validation of mothers' reports of dietary intake by four to seven year-old children. Am J Public Health 80, 1314-1317.

52. Baranowski T, Sprague D, Baranowski JH, et al. (1991) Accuracy of maternal dietary recall for preschool children. I Am Diet Assoc 91, 669-674.

53. Warren JM, Henry CJK, Livingstone MBE, et al. (2003) How well do children aged 5-7 years recall food eaten at school lunch? Public Health Nutr 6, 41-47.

54. Baranowski T, Dworkin R, Henske JC, et al. (1986) The accuracy of children's self-reports of diet: Family Health Project. J Am Diet Assoc 86, 1381-1385. 